TITLE:

\title{
Crystal plasticity finite-element simulation of work-hardening behavior in a magnesium alloy sheet under biaxial tension
}

\section{$\operatorname{AUTHOR}(S):$}

Hama, Takayuki; Takuda, Hirohiko

\section{CITATION:}

Hama, Takayuki ...[et al]. Crystal plasticity finite-element simulation of work-hardening behavior in a magnesium alloy sheet under biaxial tension. Computational Materials

Science 2012, 51(1): 156-164

ISSUE DATE:

2012-01

URL:

http://hdl.handle.net/2433/147255

\section{RIGHT:}

(c) 2011 Elsevier B.V.; この論文は出版社版でありません。引用の際には 出版社版をご確認ご利用ください。; This is not the published version. Please cite only the published version. 
Crystal Plasticity Finite-Element Simulation of Work-Hardening Behavior in a Magnesium Alloy Sheet under Biaxial Tension

Takayuki HAMA *1,*2

Hirohiko TAKUDA*1

*1 Graduate School of Energy Science, Kyoto University, Yoshida-honmachi, Sakyo-ku, Kyoto 606-8501, Japan

*2 VCAD System Research Program, RIKEN, 2-1 Hirosawa, Wako-shi, Saitama, 351-0198, Japan

Corresponding author: Takayuki HAMA

Graduate School of Energy Science, Kyoto University

Yoshida-Honmachi, Sakyo-ku, Kyoto 606-8501, Japan

TEL: $\quad+81-(0) 75-753-5418$

FAX: $\quad+81-(0) 75-753-5428$

E-mail: $\underline{\text { hama@energy.kyoto-u.ac.jp }}$ 


\section{ABSTRACT}

This paper presents the prediction of the contours of plastic work for an AZ31 magnesium alloy sheet in the first quadrant of the stress space using a rate-dependent crystal plasticity finiteelement method. The contour of plastic work was initially rather flat in the vicinity of equibiaxial tension, but bulged severely thereafter. Evidently, the shapes of the contours changed as the plastic work increased, exhibiting a differential work-hardening behavior. The variation of the relative activity of each family of slip systems was examined to investigate the mechanism of the differential work-hardening behavior. During uniaxial tension, the work-hardening was determined mainly by the basal slip in the very beginning, whereas it was determined by both the prismatic and the basal slip in the subsequent deformation. On the other hand, during equi-biaxial tension, the relative activity of the prismatic slip systems was much smaller than that under uniaxial tension, whereas the relative activity of the basal slip systems played a dominant role in the work-hardening throughout the deformation. A simple analysis revealed that it was more difficult to activate the prismatic slip systems for equi-biaxial tension than uniaxial tension in rolled magnesium alloy sheets because the two biaxial stresses tended to cancel each other thus decreasing the relative activity as the biaxial stress ratio approached unity. On the other hand, the basal slip systems were much easily activated than the prismatic slip systems although their Schmid factors were small because the shear stress required for the activation of the basal slip systems was much lower than that of the prismatic slip systems. We concluded that such differences in the activities of the slip systems eventually resulted in the differential workhardening behavior of the contour of plastic work.

Keywords: 
Magnesium alloy, Crystal plasticity, Finite-element method, Polycrystalline material, Yield locus, Schmid factor

\section{Introduction}

Reduction of the vehicle weight is an efficient approach to reduce $\mathrm{CO}_{2}$ emission and therefore, the demand for lightweight materials has been increasing for application in automobile and electrical devices [1] in order to reduce their environmental impact. Magnesium ( $\mathrm{Mg})$ alloys are the lightest materials used for structural components of vehicles and electrical devices. They not only have high specific strength and stiffness [1] but also superior functional properties such as recyclability [1, 2] and electromagnetic shielding [1-3]. Owing to these mechanical and functional characteristics, $\mathrm{Mg}$ alloys have recently received much attention.

Components made of $\mathrm{Mg}$ alloys are conventionally manufactured by die casting and thixoforming [3-5]. Recently, press forming of $\mathrm{Mg}$ alloy sheets has attracted considerable attention for the development of a wider variety of applications of structural components made of $\mathrm{Mg}$ alloys. There are many studies on the press forming of $\mathrm{Mg}$ alloy sheets, including deep drawing processes [4-8] and springback characteristics [3, 9-11].

For a wide application of press forming to $\mathrm{Mg}$ alloy sheets, their deformation characteristics should be well understood. Since Mg alloys are hexagonal close-packed (HCP) materials, $\mathrm{Mg}$ alloy sheets show mechanical properties vastly different from those of conventional structural sheet metals that have cubic structures, such as steel and aluminum alloys. Some examples are as follows: stress-strain curves of rolled $\mathrm{Mg}$ alloy sheets obtained by uniaxial tensile and 
compressive tests at room temperature are notably different [12], the stress-strain curve obtained under compression followed by tension exhibits a sigmoidal shape [12], and $\mathrm{Mg}$ alloy sheets show much stronger inelastic deformation during unloading than mild steel and aluminum alloy sheets [13-17]. Generally, it is understood that these characteristic deformations occur due to the activity of the direction-dependent $\left\{\begin{array}{llll}1 & 0 & \overline{1} & 2\end{array}\right\}$ twinning. As a strong basal texture with most of the $c$-axes aligned in the thickness direction is initially formed in the rolled $\mathrm{Mg}$ alloy sheets [12, 18], it is difficult to activate the $\left\{\begin{array}{lllll}1 & 0 & \overline{1} & 2\end{array}\right\}$ twinning under in-plane tension. The strong basal texture also leads to the poor stretch formability of $\mathrm{Mg}$ alloy sheets because the throughthickness strain does not easily develop [19, 20].

Work-hardening behavior under the biaxial stresses condition is one of the important deformation characteristics that should be understood, especially in the field of press forming. Recently, Andar et al. [21] measured experimentally the contours of plastic work in a rolled $\mathrm{AZ31} \mathrm{Mg}$ alloy sheet in the first quadrant of the stress space. The ratio of equi-biaxial stress to uniaxial stress increased and the shapes of the contours changed with the progress of plastic deformation in the plastic strain range $0.001 \leq \varepsilon^{p} \leq 0.008$, exhibiting the so-called differential work-hardening behavior [22]. Tadano [23] also showed a qualitatively similar result in pure $\mathrm{Mg}$ using crystal plasticity finite-element analysis. Choi et al. [24] and Graff et al. [25] also calculated the contours of plastic work using the crystal plasticity finite-element method and a self-consistent model, respectively. It should be emphasized that the differential work-hardening behavior appeared in the first quadrant of the stress space where it was difficult to activate the $\left\{\begin{array}{llll}1 & 0 & \overline{1} & 2\end{array}\right\}$ twinning as described above, indicating that the differential work-hardening may not be primarily due to the activity of twinning, but due to other factors. To the best of our knowledge, the causes of this behavior have not yet been understood. 
With the rapid increase in computation power, the deformation of HCP metals, including $\mathrm{Mg}$ alloys, have been studied using crystal plasticity finite-element methods [17, 25-31]. These studies showed that the stress-strain response and texture evolution of $\mathrm{Mg}$ alloys can be reasonably predicted at least for monotonic deformation.

This paper is concerned with a crystal plasticity finite-element simulation of the workhardening behavior in a rolled AZ31 $\mathrm{Mg}$ alloy sheet in the first quadrant of the stress space. Contours of plastic work are predicted using the simulation and the mechanism of the differential work-hardening behavior is examined in detail using the relative activity of slip and twinning systems and a simple analytical model.

\section{Crystal Plasticity Finite-Element Method}

The crystal plasticity finite-element method used in this study is the same as that used in Hama and Takuda [17]. The formulations used here are explained only briefly and the reader is referred to literature [17] for details.

An updated Lagrangian rate formulation is used to describe the finite deformation [32, 33]. The rate-dependent crystal plasticity model $[34,35]$ is incorporated into each Gauss point in the static finite-element method. The rate tangent modulus method [34] is used for the explicit time integration of the constitutive model. To prevent an excessive increase of the nonequilibrium between external and internal forces, the generalized $r_{\text {min }}$-strategy [36] is employed to limit the size of the increment. The maximum slip increment of each slip system in a time increment is also limited using the generalized $r_{m i n}$-strategy. 
The crystalline slip is assumed to follow Schmid's law. The slip rate $\dot{\gamma}^{(\alpha)}$ of the $\alpha$-slip system is assumed to be given by the visco-plastic power law using Schmid's resolved shear stress $\tau^{(\alpha)}$ as

$$
\frac{\dot{\gamma}^{(\alpha)}}{\dot{\gamma}_{0}}=\left|\frac{\tau^{(\alpha)}-\tau_{\mathrm{kin}}^{(\alpha)}}{\tau_{\mathrm{Y}}^{(\alpha)}}\right|^{\frac{1}{m}} \operatorname{sign}\left(\tau^{(\alpha)}-\tau_{\mathrm{kin}}^{(\alpha)}\right), \quad \tau^{(\alpha)}=\boldsymbol{s}^{(\alpha)} \cdot \boldsymbol{\sigma} \cdot \boldsymbol{m}^{(a)}, \quad \dot{\tau}_{\mathrm{Y}}^{(a)}=\sum_{\beta} q_{\alpha \beta} h\left|\dot{\gamma}^{(\beta)}\right|,
$$

where $\tau_{\text {kin }}^{(\alpha)}$ is the resolved back stress of the $\alpha$-slip system, $\tau_{\mathrm{Y}}^{(\alpha)}$ is the current slip resistance of the $\alpha$-slip system with $\tau_{\mathrm{Y}}^{(\alpha)}=\tau_{0}$ initially, $\dot{\gamma}_{0}$ is the reference strain rate, $m$ is the rate-sensitivity exponent, and $q_{\alpha \beta}$ with $\alpha=\beta$ and with $\alpha \neq \beta$ are the self- and latent- hardening moduli, respectively. The unit vectors $\boldsymbol{s}^{(\alpha)}$ and $\boldsymbol{m}^{(\alpha)}$ are the slip direction and the slip plane normal, respectively. $h$ is the rate of hardening and the hardening laws will be explained later.

The resolved back stress $\tau_{\text {kin }}^{(\alpha)}$ is given in the form [37-39]

$$
\tau_{\text {kin }}^{(\alpha)}=\boldsymbol{s}^{(\alpha)} \cdot \boldsymbol{\sigma}_{\text {kin }} \cdot \boldsymbol{m}^{(\mathrm{a})}, \quad \boldsymbol{\sigma}_{\text {kin }}=\sum_{\alpha} \Omega^{(\alpha)} \boldsymbol{p}^{(\alpha)} \text { with } \boldsymbol{p}^{(\alpha)}=\frac{1}{2}\left(\boldsymbol{s}^{(\alpha)} \otimes \boldsymbol{m}^{(\alpha)}+\boldsymbol{m}^{(\alpha)} \otimes \boldsymbol{s}^{(\alpha)}\right)
$$

where $\sigma_{\text {kin }}$ is the back stress tensor and $\Omega^{(\alpha)}$ is the scalar kinematic variable of the $\alpha$-slip system.

The evolution of $\Omega^{(\alpha)}$ due to the short-range dislocation interaction is assumed as

$$
\dot{\Omega}^{(\alpha)}=c \dot{\gamma}^{(\alpha)}-d \Omega^{(\alpha)}\left|\dot{\gamma}^{(\alpha)}\right|,
$$

where $c$ and $d$ are the direct hardening and dynamic recovery coefficients, respectively. Since neither the unloading nor the reverse loading is dealt with in this study, the effect of the back stress on the simulation result may be negligible. Therefore, the parameters $c$ and $d$ are set to zero in this study. 
Following Graff et al. [25], three families of slip systems-basal $<a>$ slip, prismatic $<a>$ slip, and pyramidal-2 $\left\langle\mathrm{a}+\mathrm{c}>\right.$ slip-and one family of $\left\{\begin{array}{llll}1 & 0 & \overline{1} & 2\end{array}\right\}$ twinning systems are used to model the mechanical behavior of a Mg alloy sheet. There are 3 basal, 3 prismatic, 6 pyramidal-2, and 6 twinning systems. Table 1 shows the slip/twinning plane normal and slip direction vectors for these systems.

Depending on the family of slip systems, two evolution laws are used for the rate of hardening $h$ in the forms [25]

$$
\begin{aligned}
& h=h_{0}, \\
& h=h_{0}\left(1-\frac{\tau_{0}}{\tau_{\infty}}\right) \exp \left(-\frac{h_{0} \bar{\gamma}}{\tau_{\infty}}\right),
\end{aligned}
$$

where $\bar{\gamma}$ is the cumulative shear strain on all the slip systems and is given by

$$
\bar{\gamma}=\sum_{\alpha} \int\left|\dot{\gamma}^{(\alpha)}\right| \mathrm{d} t
$$

Linear hardening (Eq. (4)) is assumed for the basal slip, and Voce hardening (Eq. (5)) for the prismatic and pyramidal-2 slip.

As for the $\left\{\begin{array}{llll}1 & 0 & \overline{1} & 2\end{array}\right\}$ twinning model, the following statistical approach [17, 26, 29, 31, $40]$ is used. Twinning is assumed to have a polar character, where each system can be activated only by the tension of the $c$-axis. The shear strain that arises in a grain due to twinning is assumed to be given by $\bar{\gamma}_{\text {twin }}^{(\alpha)}=f^{(\alpha)} \gamma_{\text {ref }}$, where $f^{(\alpha)}$ is the volume fraction of the $\alpha$-twinning system, $\gamma_{\text {ref }}$ is the shear strain when the grain is twined entirely, and $\bar{\gamma}_{\text {twin }}^{(\alpha)}$ is the cumulative shear strain on the $\alpha$-twinning system. Since this shear is similar to the shear caused by an activated slip system, the shear strain rate induced by twinning can be calculated in a way similar to that 
used for slip deformation calculation using Eq.(1). Equation (4) is used for the rate of hardening $h$ of the twinning systems.

The lattice rotation due to twinning is modeled as follows. A pseudo-random threshold value $f_{\mathrm{th}}^{(\alpha)}$ is initially determined between 0.3 and 1.0 for each twinning system $[17,26,29,31]$. During the calculation, the volume fraction of the $\alpha$-twinning system $f^{(\alpha)}$ is compared with $f_{\mathrm{th}}^{(\alpha)}$ at each time increment, and if $f^{(\alpha)} \geq f_{\mathrm{th}}^{(\alpha)}$ is satisfied, the entire grain is rotated to a specific twinning orientation. The lattice rotation tensor due to twinning $\boldsymbol{R}^{\mathrm{tw}}$ is given in the form

$$
\boldsymbol{R}^{t w}=2 \boldsymbol{m}^{(\alpha)} \otimes \boldsymbol{m}^{(\alpha)}-\boldsymbol{I}
$$

where $\boldsymbol{I}$ is the unit tensor and $\boldsymbol{m}^{(\alpha)}$ is the twinning plane normal vector for the $\alpha$-twinning system. Using the twinning model described above, $\gamma_{\text {ref }}$ can also be viewed as a material parameter. In this study, we assume that the detwinning can be ignored because neither unloading nor reverse loading is dealt with here as described above.

The material parameters used in Eqs.(1), (4), and (5) and $\gamma_{\text {ref }}$ will be determined in the next section.

\section{Simulation procedures}

\subsection{Material Modeling}

The simulation procedures used in this study were basically the same as those used by Hama and Takuda [17]. Figure 1 shows the schematic of the finite-element model. The cubic model was divided into ten uniform eight-node isoparametric brick elements using selective reduced integration in each direction. Initial crystallographic orientations of the eight Gauss integration points in an element were the same; thus the number of initial crystallographic orientations of the 
model was $10 \times 10 \times 10=1000$. The initial (0001) pole figure of the model is shown in Fig. 2 . The initial crystallographic orientations were artificially created using the procedure proposed by Mayama et al. [29] to simulate the rolling texture of Mg alloy sheets [19, 25, 41]. It should be noted that the lattice orientation was updated independently at each Gauss integration point.

We employed the above finite-element model because its number of initial crystallographic orientations was sufficient to predict the mechanical behaviors of $\mathrm{Mg}$ alloys [17, 25, 31, 42]. The effects of mesh discretization and initial crystallographic orientations on the stress-strain curve obtained by a uniaxial tensile test were examined in Hama and Takuda [17]. These results showed that our present model is acceptable for the investigation of deformation at least up to a strain of about $3 \%$. The effect of mesh discretization on the contour of plastic work will be discussed later.

In the simulation, the $x$-, $y$-, and $z$-axes in Fig. 1 were defined to be the rolling direction (RD), transverse direction (TD), and normal direction (ND), respectively; thus the majority of $c$-axes tended to align in the $z$ direction (ND).

\subsection{Boundary conditions}

The contour of plastic work was calculated using the following procedure employed in Andar et al. [21]. Uniaxial tension in RD was selected as the reference condition. The uniaxial stress $\sigma_{0}$ and the plastic work per unit volume $W^{\mathrm{p}}$ were calculated at various uniaxial plastic strains $\varepsilon_{R D}^{p}$, which were given by integrating the plastic strain rate $\boldsymbol{D}^{\mathrm{P}}$ during the uniaxial tension. The plastic work $W^{\mathrm{p}}$ is given in the form

$$
W^{p}=\int \boldsymbol{\sigma} \cdot \boldsymbol{D}^{\mathrm{p}} \mathrm{dt}, \quad \text { with } \boldsymbol{D}^{\mathrm{p}}=\sum_{\alpha=1}^{N} \dot{\gamma}^{(\alpha)} \frac{1}{2}\left(\boldsymbol{s}^{(\alpha)} \otimes \boldsymbol{m}^{(\alpha)}+\boldsymbol{m}^{(\alpha)} \otimes \boldsymbol{s}^{(\alpha)}\right),
$$


where $N$ is the total number of slip and twinning systems in a grain. Then, the simulations of biaxial tension were carried out under various proportional stress paths and the biaxial stresses $\sigma_{\mathrm{RD}}$ and $\sigma_{\mathrm{TD}}$ were determined at the same plastic work. In the present study, the prescribed biaxial stress ratios were $\sigma_{\mathrm{TD}}: \sigma_{\mathrm{RD}}=1: 0,8: 1,3: 1,2: 1,1.5: 1,1: 1,1: 1.5,1: 2,1: 3,1: 8$, and 0:1. Consequently, the contours of plastic work could be obtained for various $\varepsilon_{R D}^{p}$ by plotting the obtained stresses in the principal stress space. The contour of plastic work was calculated up to $\varepsilon_{R D}^{p}=1.56 \%$ in this study. It should be noted that the plastic work $W^{\mathrm{p}}$ was not exactly zero, even within the elastic range in the stress-strain curve because of the rate-dependent model of crystal plasticity.

The following boundary conditions were used to simulate the contour of plastic work under proportional stress paths. The planes $x=0, y=0$, and $z=0$ of the model (Fig. 1) were fixed in the $x, y$, and $z$ directions, respectively, according to the assumption of plane symmetry. Small displacement increments were given to the planes $x=l$ and $y=l$ using the following procedure such that pseudo-proportional stress paths were achieved. The simulation with $\sigma_{\mathrm{TD}} / \sigma_{\mathrm{RD}}=\alpha(\leq 1)$ was assumed for the following.

(i) Small displacement increments are given to the plane $x=l$.

(ii) Then, small displacement increments are also given to the plane $y=l$ by fixing the plane $x=l$.

(iii) If $\sigma_{\mathrm{TD}} / \sigma_{\mathrm{RD}} \approx \alpha$ is satisfactorily achieved, go to (i). Otherwise, repeat (ii).

If $\alpha>1, x$ and $y$ are opposite. The above procedure is repeated until the plastic work of $\varepsilon_{R D}^{p}=$ $1.56 \%$ is obtained. The validity of the procedure will be discussed in the following sections. 
Young's modulus $E=42 \mathrm{GPa}$ and Poisson's ratio $v=0.3$ were assumed. The rate-sensitivity exponent was set to $m=0.02$. The choice of $m$ has been discussed in Hama and Takuda [17]. The reference strain rate was set to $\dot{\gamma}_{0}=0.001 \mathrm{~s}^{-1}$. Following Graff et al. [25], the self-hardening parameters were set as 1 and the latent-hardening parameters shown in Table 2 were adopted. $\gamma_{\text {ref }}$ was set to 0.1. The hardening parameters used were the same as those used in Hama and Takuda [17]. The parameters used in equations (4) and (5) were identified by trial and error to achieve reasonable fits with the experimental stress-strain curves obtained from the uniaxial tensile and compressive tests for the AZ31 Mg alloy sheet with a thickness of $0.8 \mathrm{~mm}$. The identification of the hardening parameters has been explained in detail in Hama and Takuda [17]. The determined parameters are shown in Table 3. The simulated and experimental stress-strain curves are shown in Fig. 3. Although the simulated curve under monotonic compression was different from that of the experiment at strains higher than -0.07 , this difference might be negligible because the deformations until only $\varepsilon_{R D}^{p}=1.56 \%$ were analyzed in this study, as described above.

As already explained in Hama and Takuda [17], we could not guarantee that these parameters are in best agreement and are unique for this material. In fact, we have already showed that a very similar stress-strain curve shown in Fig. 3 can be obtained using another set of hardening parameters [43]. Nonetheless, the activities of the slip and twinning systems under monotonic tension obtained using this set of hardening parameters were in good agreement qualitatively with the present results that are shown below. Therefore, we concluded that the following discussion was acceptable regardless of the set of hardening parameters used.

4. Results and discussion

4.1 Contour of plastic work 
First, the linearity of the stress path during deformations is examined to verify the simulation procedures adopted in this study. Figure 4 shows the evolutions of $\sigma_{\mathrm{RD}}$ and $\sigma_{\mathrm{TD}}$ for the prescribed biaxial stress ratios up to $\varepsilon_{R D}^{p}=1.56 \%$. Almost linear stress paths are achieved with the designated gradients regardless of the stress ratios, indicating that the simulation procedures are acceptable for the calculation of the contours of plastic work.

Figure 5 shows the contours of plastic work in the uniaxial plastic strain range $0.08 \% \leq \varepsilon_{R D}^{p} \leq$ $1.56 \%$. The contours normalized by the uniaxial stress $\sigma_{0}$ and the theoretical yield locus calculated using the von Mises isotropic yield function are shown in Fig. 6. Overall, the contours are almost symmetric in $x$ and $y$ directions. From the beginning of plastic deformation $\left(\varepsilon_{R D}^{p}=\right.$ $0.08 \%$ ) to $\varepsilon_{R D}^{p}=0.16 \%$, the contour is flattened in the vicinity of $\sigma_{\mathrm{TD}} / \sigma_{\mathrm{RD}}=1$, at which the stresses are smaller than the theoretical locus (Fig. 6 (a)). Subsequently, in the uniaxial plastic strain range $0.16 \% \leq \varepsilon_{R D}^{p} \leq 0.3 \%$, the work-hardening rates in the vicinity of $\sigma_{\mathrm{TD}} / \sigma_{\mathrm{RD}}=1$ become larger than those in the vicinity of $\sigma_{\mathrm{TD}} / \sigma_{\mathrm{RD}}=0$ and the contour bulges severely. At the same time, the stresses in the vicinity of $\sigma_{\mathrm{TD}} / \sigma_{\mathrm{RD}}=1$ become larger than the theoretical locus. Clearly, the shapes of the contours change as $\varepsilon_{R D}^{p}$ increases, exhibiting a differential workhardening behavior in the simulation result. Then, at $\varepsilon_{R D}^{p}$ higher than $0.3 \%$, the work-hardening rates in the vicinity of $\sigma_{\mathrm{TD}} / \sigma_{\mathrm{RD}}=1$ become small again and the shapes of the contours do not change thereafter. The above trends observed in the simulation results are qualitatively in good agreement with those of the experimental results reported by Andar et al. [21], demonstrating that the present simulation results are qualitatively acceptable.

To examine the effect of mesh discretization on the contour of plastic work, we prepared two other finite-element models, in which the cubic region is divided in each direction into seven 
uniform eight-node brick elements using selective reduced integration and into seven uniform twenty-node brick elements using reduced integration, respectively. Figure 7 shows the normalized contours in the uniaxial plastic strain range $0.08 \% \leq \varepsilon_{R D}^{p} \leq 0.8 \%$ obtained using the two models. Although the rate of expansion of the contour is slightly different in the results obtained with twenty-node brick elements (Fig. 7 (b)), the trends in the change of the contour shapes are almost independent of the mesh discretization.

\subsection{Relative Activity of Slip Systems}

In this subsection, variation of the relative activity of each family of slip and twinning systems is used to investigate the mechanism of the differential work-hardening behavior. The relative activity is the relative contribution of each family of slip and twinning systems to the plastic deformation. The relative activity of each family of slip and twinning systems $i$ is given in the form [44]

$$
r_{i}=\frac{\sum_{p=1}^{n} \sum_{\alpha=1}^{k}\left|\Delta \gamma^{(p, \alpha)}\right|}{\sum_{s=1}^{n} \sum_{\beta=1}^{N}\left|\Delta \gamma^{(s, \beta)}\right|},
$$

where the numerator is the plastic strain increment contributed by the family of slip or twinning systems $i$, summed over all the grains. $k$ is the number of slip or twinning systems of the family $i$ and $n$ is the number of grains. The denominator is the plastic strain increment contributed by all the families, summed over all the grains.

The variations of the relative activities of each family of slip and twinning systems as functions of the plastic work obtained using the biaxial stress ratios $\sigma_{\mathrm{TD}} / \sigma_{\mathrm{RD}}=0,1 / 8,1 / 3,1 / 2$, 2/3, and 1are shown in Fig. 8. The correlation between the plastic work and the uniaxial plastic 
strain $\varepsilon_{R D}^{p}$ is also shown in Fig. 8 (a). The variation of the relative activities during the uniaxial tension $\left(\sigma_{\mathrm{TD}} / \sigma_{\mathrm{RD}}=0\right)$ (Fig. $\left.8(\mathrm{a})\right)$ is as follows. The relative activity of the basal slip systems is predominant at the very beginning of the plastic deformation. Shortly after that the relative activity of the prismatic slip systems starts increasing rapidly and at the same time, the relative activity of the basal slip systems decreases. During the middle stage of plastic deformation, both the prismatic slip and the basal slip systems are activated and the relative activity of the prismatic slip systems is more predominant. The relative activities of the pyramidal-2 slip and twinning systems are negligible throughout the deformation simulated in this study.

The overall transitions of the relative activity are qualitatively similar regardless of the biaxial stress ratio. On the other hand, from a quantitative point of view, the transitions change as the biaxial stress ratio increases in the following manner: the initial plastic strain range where only the basal slip is activated becomes larger, the relative activity of the prismatic slip becomes smaller, and the relative activity of the pyramidal-2 slip becomes larger. Moreover, the initial gradient when the relative activity of the prismatic slip systems starts increasing becomes smaller as the biaxial stress ratio increases. Furthermore, the relative activities during the equi-biaxial tension $\left(\sigma_{\mathrm{TD}} / \sigma_{\mathrm{RD}}=1\right)$ (Fig. 8 (f)) are as follows. The initial plastic strain range where only the basal slip is activated seems to be the largest among all the biaxial ratio conditions. The prismatic slip and pyramidal-2 slip systems then start getting activated gradually. Since the relative activity of the prismatic slip and pyramidal-2 slip systems is much smaller than that of the prismatic slip during uniaxial tension at the middle stage, the relative activity of the basal slip systems remains predominant. Interestingly, the relative activity of the prismatic slip systems is smaller than that of the pyramidal-2 slip systems. The relative activity of the twinning systems is negligible throughout the deformation. 
From the above results, the correlation between the work-hardening behavior and the relative activity of the slip systems can be explained as follows. The contours of plastic work are flattened in the vicinity of $\sigma_{\mathrm{TD}} / \sigma_{\mathrm{RD}}=1$ at small plastic strains because the initial plastic strain range where only the basal slip is activated becomes larger as the biaxial stress ratio increases; thus the stresses in the vicinity of $\sigma_{\mathrm{TD}} / \sigma_{\mathrm{RD}}=1$ are smaller than those in the vicinity of $\sigma_{\mathrm{TD}} / \sigma_{\mathrm{RD}}=$ 0. Thereafter, the nonbasal slip systems start getting activated. Since the work-hardening rates of the nonbasal slip systems are much larger than those of the basal slip systems (Table 3), the work-hardening rate in the macroscopic stress-strain curve would also increase. This increase in the work-hardening rate is clearer in the vicinity of $\sigma_{\mathrm{TD}} / \sigma_{\mathrm{RD}}=1$ because the plastic strain range where only the basal slip is activated at the beginning is noticeable as described before. Moreover, the relative activity of the pyramidal-2 slip systems, whose hardening parameters are larger than those of the prismatic slip systems, becomes larger as the biaxial stress ratio increases. As a consequence, the increase in the work-hardening rate is larger in the vicinity of $\sigma_{\mathrm{TD}} / \sigma_{\mathrm{RD}}=1$ than that of $\sigma_{\mathrm{TD}} / \sigma_{\mathrm{RD}}=0$; hence the contours of plastic work bulge severely in the vicinity of $\sigma_{\mathrm{TD}} / \sigma_{\mathrm{RD}}=1$

Summarizing the above results, we conclude that the differential work-hardening in a rolled $\mathrm{Mg}$ alloy sheet is due to the facts that the slip systems that govern the work-hardening are notably different depending on the biaxial stress ratio and that the variation of the relative activity is also different depending on the biaxial stress ratio.

It is known that twin boundaries interact significantly with dislocations and act as barriers to slip and twinning deformation, causing work-hardening by pile-ups of the dislocations [45-48]. Although this phenomenon was not taken into consideration in the above simulation, the differential work-hardening behavior in a rolled $\mathrm{Mg}$ alloy sheet was appropriately predicted in 
the present simulation. This may be because the activity of the twinning systems was negligible in the first quadrant of the stress space as shown in Fig. 8; hence the effect of this interaction was less important. On the other hand, this phenomenon would play an important role in the workhardening under in-plane compression during which the activity of the twinning systems is much larger. Therefore it is presumed that this phenomenon should be taken into consideration when simulating the contour of plastic work in other quadrants of the stress space.

\subsection{Mechanism of Differential Work-Hardening}

Figure 9 shows the relationships between the relative activity and the biaxial stress ratio at $\varepsilon_{R D}^{p}=0.2 \%$ and $1.0 \%$. The relative activity of the prismatic slip systems at $\sigma_{\mathrm{TD}} / \sigma_{\mathrm{RD}}=0$ (uniaxial tension) is the largest among all the biaxial ratio conditions for both plastic strains. In addition, it is the largest among the activated systems at $\varepsilon_{R D}^{p}=1.0 \%$ (Fig. 9 (b)) and $\sigma_{\mathrm{TD}} / \sigma_{\mathrm{RD}}=0$ (uniaxial tension), as described before. However, the relative activity of the prismatic slip systems decreases as $\sigma_{\mathrm{TD}} / \sigma_{\mathrm{RD}}$ approaches unity and is the smallest at $\sigma_{\mathrm{TD}} / \sigma_{\mathrm{RD}}=1$ (equi-biaxial tension) for both plastic strains. On the other hand, the relative activities of other slip systems, i.e., the basal slip systems for $\varepsilon_{R D}^{p}=0.2 \%$ and the basal slip and pyramidal- 2 slip systems for $\varepsilon_{R D}^{p}=1.0 \%$, increase as $\sigma_{\mathrm{TD}} / \sigma_{\mathrm{RD}}$ approaches unity as described above. It is presumed from these results that one of the main reasons for the differential work-hardening behavior is that the relative activity of the prismatic slip systems decreases as the biaxial stress ratio approaches unity. To examine the mechanism of the change in the activity of the prismatic slip systems, the following simple analytical model is used. To simplify the model, an idealized HCP crystal structure whose $c$-axis trends exactly in the sheet normal direction is considered as shown in Fig. 10, although the 
directions of the $c$-axes in rolled $\mathrm{Mg}$ alloy sheets in fact exhibit some variation from the sheet normal direction as shown in Fig. 2. Assuming that biaxial stresses $\sigma_{\mathrm{RD}}$ and $\sigma_{\mathrm{TD}}$ are acting on the crystal structure in the $x$ and $y$ directions respectively, the Schmid's resolved shear stress of an arbitrary slip system can be expressed in the form

$$
\begin{aligned}
\tau_{\text {slip }} & =\boldsymbol{m} \cdot \boldsymbol{\sigma} \cdot \boldsymbol{s} \\
& =\left\{\begin{array}{lll}
m_{x} & m_{y} & m_{z}
\end{array}\right\}\left[\begin{array}{ccc}
\sigma_{\mathrm{RD}} & 0 & 0 \\
0 & \sigma_{\mathrm{TD}} & 0 \\
0 & 0 & 0
\end{array}\right] \mid\left\{\begin{array}{c}
s_{x} \\
\left\{s_{y}\right.
\end{array}\right\} \\
& =m_{x} s_{x} \sigma_{\mathrm{RD}}+m_{y} s_{y} \sigma_{\mathrm{TD}},
\end{aligned}
$$

where $m_{i}$ and $s_{i}$ are the components of the unit vectors $\boldsymbol{s}$ and $\boldsymbol{m}$ of this slip system, which refer to the $x$-y coordinate system in Fig. 10. Using the angles $\theta$ and $\varphi$ shown in Fig. 10, $s$ and $\boldsymbol{m}$ of an arbitrary prismatic slip system are given by

$$
\boldsymbol{m}=\left\{\begin{array}{c}
\cos \theta \\
\sin \theta \\
0
\end{array} \mid, \quad \boldsymbol{s}=\left\{\begin{array}{c}
\cos \varphi \\
\sin \varphi \\
\sin \\
0
\end{array}\right\} .\right.
$$

Introducing Eq. (11) in Eq.(10) yields the resolved shear stress of the prismatic slip $\tau_{\text {pris }}$ given in the form

$$
\tau_{\text {pris }}=\left(\sigma_{\mathrm{RD}}-\sigma_{\mathrm{TD}}\right) \sin \varphi \cos \varphi
$$

Equation (12) shows that the magnitudes of the Schmid factors for $\sigma_{\mathrm{TD}}$ and $\sigma_{\mathrm{RD}}$ are the same but their signs are opposite. Therefore, $\tau_{\text {pris }}$ becomes smaller as the biaxial stress ratio $\sigma_{\mathrm{TD}} / \sigma_{\mathrm{RD}}$ approaches unity, decreasing the activity of the prismatic slip systems. It should be noted that the activity of the prismatic slip systems may be larger in real rolled $\mathrm{Mg}$ alloy sheets than that expected from Eq. (12) because the $c$-axes are not exactly aligned in the sheet normal direction, but a similar trend would be observed. 
On the other hand, the basal slip systems are much easily activated than the prismatic slip systems although the Schmid factor of the basal slip systems is small in rolled Mg alloy sheets because of the strong basal texture. This is because the shear stress required for the activation of the basal slip systems is much lower than that of the prismatic slip systems as shown in Table 3 . Hence, the activity of the basal slip systems is predominant at the beginning of the plastic deformation regardless of the biaxial stress ratio. Since it becomes more difficult to activate the prismatic slip systems as $\sigma_{\mathrm{TD}} / \sigma_{\mathrm{RD}}$ approaches unity as understood from Eq. (12), the initial plastic strain range where only the basal slip is activated becomes larger and the relative activity of the basal slip systems increases as $\sigma_{\mathrm{TD}} / \sigma_{\mathrm{RD}}$ approaches unity.

The reason why the relative activity of the pyramidal-2 slip systems increases as $\sigma_{\mathrm{TD}} / \sigma_{\mathrm{RD}}$ approaches unity for $\varepsilon_{R D}^{p}=1.0 \%$ can be explained as follows. Table 4 shows the Schmid factors $m_{x} s_{x}$ and $m_{y} s_{y}$ in Eq. (10) of the pyramidal-2 slip systems. It is clear that the two Schmid factors do not cancel each other as those in the prismatic slip systems. This is because the slip planes tilt from the $c$-axis. Furthermore, the biaxial stress ratio, at which the resolved shear stress of the pyramidal-2 slip $\tau_{\text {pyram }}$ becomes zero, is different depending on the slip system, and it is even negative in some slip systems, indicating that the family of the pyramidal-2 slip systems can always be activated in the first quadrant of the stress space. As a consequence, the activity of the pyramidal-2 slip systems, instead of the prismatic slip systems, increases as $\sigma_{\mathrm{TD}} / \sigma_{\mathrm{RD}}$ approaches unity. The activity of the pyramidal-2 slip systems is negligible for $\sigma_{\mathrm{TD}} / \sigma_{\mathrm{RD}}=0$ because the prismatic slip systems are still easy to be activated and the shear stress required for the activation of the pyramidal-2 slip systems is much larger than that of the prismatic slip systems. Similarly, the activity of the pyramidal- 2 slip systems is negligible at $\varepsilon_{R D}^{p}=0.2 \%$ regardless of the biaxial stress ratio because the applied tensile stresses are too small for the pyramidal-2 slip systems to 
be activated. We conclude that such differences in the activities of the slip systems eventually result in the differential work-hardening behavior.

\section{Conclusion}

The contour of plastic work for an AZ31 magnesium alloy sheet was predicted using ratedependent crystal plasticity finite-element method. The mechanism of the differential workhardening behavior was examined in detail using the relative activity of each family of slip systems and a simple analytical model. The results obtained in this study are as follows:

(1) The contour of plastic work obtained in the simulation clearly exhibits a differential workhardening behavior as follows. The contour is initially rather flattened in the vicinity of equibiaxial tension, but severely bulges at the middle stage. This trend is qualitatively in good agreement with the experimental result reported in literature [21].

(2) From the variations of the relative activity, the mechanism of the differential work-hardening behavior can be explained as follows. Only the basal slip systems are activated at the beginning of the plastic deformation regardless of the biaxial stress ratio, but the plastic strain range where only the basal slip is activated becomes larger as the biaxial stress ratio approaches unity. As a result, the stresses in the vicinity of equi-biaxial tension are determined solely from the basal slip at small plastic strains; they yield flattened contours of plastic work in the vicinity of equi-biaxial tension at small plastic strains. Thereafter, the nonbasal slip systems start getting activated during the middle stage. The relative activity of the pyramidal-2 slip systems in the vicinity of equibiaxial tension is larger than that in the vicinity of uniaxial tension. Since the hardening parameters of the pyramidal-2 slip systems are larger than those of the prismatic slip systems, the increase in the work-hardening rate in the vicinity of equi-biaxial tension is larger than that in the 
vicinity of uniaxial tension, yielding severely bulging contours of plastic work in the vicinity of equi-biaxial tension.

(3) One of the main reasons for the differential work-hardening behavior is that the relative activity of the prismatic slip systems decreases as the biaxial stress ratio approaches unity. A simple analysis reveals that the prismatic slip systems are more difficult to activate under equibiaxial tension than under uniaxial tension in rolled magnesium alloy sheets because the two biaxial stresses tend to cancel each other. On the other hand, the basal slip systems are comparatively easily activated than the prismatic slip systems although their Schmid factors are small in rolled $\mathrm{Mg}$ alloy sheets because of the strong basal texture. This is because the shear stress required for the activation of the basal slip systems is much lower than that of the prismatic slip systems. Similarly, the Schmid factors of the pyramidal-2 slip systems show that the pyramidal-2 slip systems are much easily activated than the prismatic slip systems in the vicinity of equi-biaxial tension although the shear stress required for the activation of the pyramidal-2 slip systems is higher; thus the relative activity of the pyramidal-2 slip systems increases instead of that of the prismatic slip systems as the biaxial stress ratio approaches unity during the middle stage. We conclude that such differences in the activities of the slip systems eventually result in the differential work-hardening behavior of the contour of plastic work.

\section{Acknowledgements}

This work was partially supported by KAKENHI (23760697: Grant-in-Aid for Young Scientist (B)) in Japan and the Amada Foundation for metal work technology. The authors wish to acknowledge Professor T. Kuwabara of Tokyo University of Agriculture and Technology for his helpful advice on the contour of plastic work. 


\section{REFERENCES}

[1] B.L. Mordike, T. Ebert, Materials Science and Engineering A 302 (2001) 37-45.

[2] Y. Chino, T. Furuta, M. Hakamada, M. Mabuchi, Journal of Materials Research 41 (2006) 3229-3232.

[3] H.J. Kim, S.C. Choi, K.T. Lee, H.Y. Kim, Materials Transactions 49 (2008) 1112-1119.

[4] J. Kaneko, M. Sugamata, J. JILM 54 (2004) 484-492 (in Japanese).

[5] Y.S. Lee, M.C. Kim, S.W. Kim, Y.N. Kwon, S.W. Choi, J.H. Lee, Journal of Materials Processing Technology 187-188 (2007) 103-107.

[6] E. Doege, K. Droder, Journal of Materials Processing Technology 115 (2001) 14-19.

[7] F.K. Chen, T.B. Huang, C.K. Chang, International Journal of Machine Tools \& Manufacture 43 (2003) 1553-1559.

[8] S. Lee, Y.H. Chen, J.Y. Wang, Journal of Materials Processing Technology 124 (2002) 1924.

[9] F.K. Chen, T.B. Huang, Journal of Materials Processing Technology 142 (2003) 643-647.

[10] C. Bruni, A. Forcellese, F. Gabrielli, M. Simoncini, Journal of Materials Processing Technology 177 (2006) 373-376.

[11] T. Hama, Y. Kariyazaki, K. Ochi, H. Fujimoto, H. Takuda, Materials Transactions 51 (2010) 685-693.

[12] X.Y. Lou, M. Li, R.K. Boger, S.R. Agnew, R.H. Wagoner, International Journal of Plasticity 23 (2007) 44-86.

[13] C.H. Cáceres, T. Sumitomo, M. Veidt, Acta Materialia 51 (2003) 6211-6218. 
[14] G.E. Mann, T. Sumitomo, C.H. Cáceres, J.R. Griffiths, Materials Science and Engineering A 456 (2007) 138-146.

[15] Y. Li, M. Enoki, Materials Transactions 49 (2008) 1800-1805.

[16] O. Muránsky, D.G. Carr, P. Sittner, E.C. Oliver, International Journal of Plasticity 25 (2009) $1107-1127$.

[17] T. Hama, H. Takuda, International Journal of Plasticity 27 (2011) 1072-1092.

[18] Y. Chino, J.S. Lee, K. Sassa, A. Kamiya, M. Mabuchi, Materials Letters 60 (2006) 173176.

[19] Y. Chino, K. Sassa, A. Kamiya, M. Mabuchi, Materials Science and Engineering A 473 (2008) 195-200.

[20] J. Koike, Metallurgical and Materials Transactions A 36 (2005) 1689-1696.

[21] M.O. Andar, D. Steglich, T. Kuwabara, Proc. AMPT '10, Paris, France, October, 2010, pp. 75-80.

[22] R. Hill, J.W. Hutchinson, Journal of applied mechanics_-Transactions of the ASME 59 (1992) S1-S9.

[23] Y. Tadano, International Journal of Mechanical Sciences 52 (2010) 257-265.

[24] S.-H. Choi, D.H. Kim, H.W. Lee, B.S. Seong, K. Piao, R. Wagoner, Materials Science and Engineering A 526 (2009) 38-49.

[25] S. Graff, W. Brocks, D. Steglich, International Journal of Plasticity 23 (2007) 1957-1978.

[26] A. Staroselsky, L. Anand, International Journal of Plasticity 19 (2003) 1843-1864.

[27] A. Prakash, S.M. Weygand, H. Riedel, Computational Materials Science 45 (2009) 744-750.

[28] W. Tang, S. Zhang, Y. Peng, D. Li, Computational Materials Science 46 (2009) 393-399. 
[29] T. Mayama, K. Aizawa, Y. Tadano, M. Kuroda, Computational Materials Science 47 (2009) 448-455.

[30] T. Walde, H. Riedel, Acta Materialia 55 (2007) 867-874.

[31] T. Mayama, M. Noda, R. Chiba, M. Kuroda, International Journal of Plasticity 2011 in press.

[32] M. Kawka, A. Makinouchi, Journal of Materials Processing Technology 50 (1995) 105-115.

[33] T. Hama, T. Nagata, C. Teodosiu, A. Makinouchi, H. Takuda, International Journal of Mechanical Sciences 50 (2008) 175-192.

[34] D. Pierce, R.J. Asaro, A. Needleman, Acta Metallurgica 31 (1983) 1951-1976.

[35] R.J. Asaro, A. Needleman, Acta Metallurgica 33 (1985) 923-953.

[36] Y. Yamada, N. Yoshimura, T. Sakurai, International Journal of Mechanical Sciences 10 (1968) 343-354.

[37] J. Harder, International Journal of Plasticity 15 (1999) 605-624.

[38] C.L. Xie, S. Ghosh, M. Groeber, Journal of Engineering Materials and TechnologyTransactions of the ASME 126 (2004) 339-352.

[39] E. Nakamachi, N.N. Tam, H. Morimoto, International Journal of Plasticity 23 (2007) 450489.

[40] P. Van Houtte, Acta Metallurgica 26 (1978) 591-604.

[41] S.R. Agnew, Ö. Duygulu, International Journal of Plasticity 21 (2005) 1161-1193.

[42] O. Diard, S. Leclercq, G. Rousselier, G. Cailletaud, International Journal of Plasticity 21 (2005) 691-722.

[43] T. Hama, H. Fujimoto, H. Takuda, Proc. NUMIFORM '10, Pohang, Korea, June, 2010, pp. 802-809, CD-ROM. 
[44] C.N. Tomé, R.A. Lebensohn, U.F. Kocks, Acta Metallurgica et Materialia 39 (1991) 26672680.

[45] J. W. Christian, S. Mahajan, Progress in Materials Science 39 (1995), 1-157.

[46] A. Serra, D. J. Bacon, Acta Metallurgica et Materialia 43 (1995), 4465-4481.

[47] A. Serra, D. J. Bacon, R. C. Pond, Metallurgical and Materials Transactions A 33 (2002), 809-812.

[48] K. N. Braszczynska-Malik, L. Litynska, W. Baliga, Journal of Microscopy, 224 (2006), 1517.

\section{Figure Captions}

Fig. 1 Finite-element model used in the simulation.

Fig. 2 (0001) pole figure used in the simulation model with rolling texture. 
Fig. 3 Stress-strain curves under uniaxial tension and compression obtained by experiment and simulation.

Fig. 4 Stress paths during deformations up to $\varepsilon_{R D}^{p}=1.56 \%$.

Fig. 5 Contours of plastic work obtained from simulation. (a) $0.08 \% \leq \varepsilon_{R D}^{p} \leq 0.8 \%$ and (b) $0.8 \% \leq \varepsilon_{R D}^{p} \leq 1.56 \%$

Fig. 6 Normalized contours of plastic work obtained from simulation. (a) $0.08 \% \leq \varepsilon_{R D}^{p} \leq 0.8 \%$ and (b) $0.8 \% \leq \varepsilon_{R D}^{p} \leq 1.56 \%$.

Fig. 7 Normalized contours of plastic work obtained using the two other finite-element models. (a) Seven uniform eight-node brick elements and (b) seven uniform twenty-node brick elements.

Fig. 8 Variations of relative activities as a function of plastic work. (a) $\sigma_{\mathrm{TD}} / \sigma_{\mathrm{RD}}=0$, (b) $\sigma_{\mathrm{TD}} / \sigma_{\mathrm{RD}}=1 / 8$, (c) $\sigma_{\mathrm{TD}} / \sigma_{\mathrm{RD}}=1 / 3$, (d) $\sigma_{\mathrm{TD}} / \sigma_{\mathrm{RD}}=1 / 2$, (e) $\sigma_{\mathrm{TD}} / \sigma_{\mathrm{RD}}=2 / 3$ and (f) $\sigma_{\mathrm{TD}} / \sigma_{\mathrm{RD}}=1$.

Fig. 9 Correlations between relative activity and biaxial stress ratio. (a) $0.08 \% \leq \varepsilon_{R D}^{p} \leq 0.8 \%$ and (b) $0.8 \% \leq \varepsilon_{R D}^{p} \leq 1.56 \%$.

Fig. 10 Simple analytical model of HCP crystal structure on which biaxial stresses are acting.

\section{Table Captions}

Table 1. Plane normal and slip direction vectors of slip and twinning systems used in the present study.

Table 2. Latent-hardening parameters $q_{\alpha \beta}$ used in the present study (Graff et al. [25]).

Table 3. Calibrated material parameters in Eqs. (4) and (5).

Table 4. The Schmid factors $m_{x} s_{x}$ and $m_{y} s_{y}$ in eq.(10) of the pyramidal-2 slip systems. 


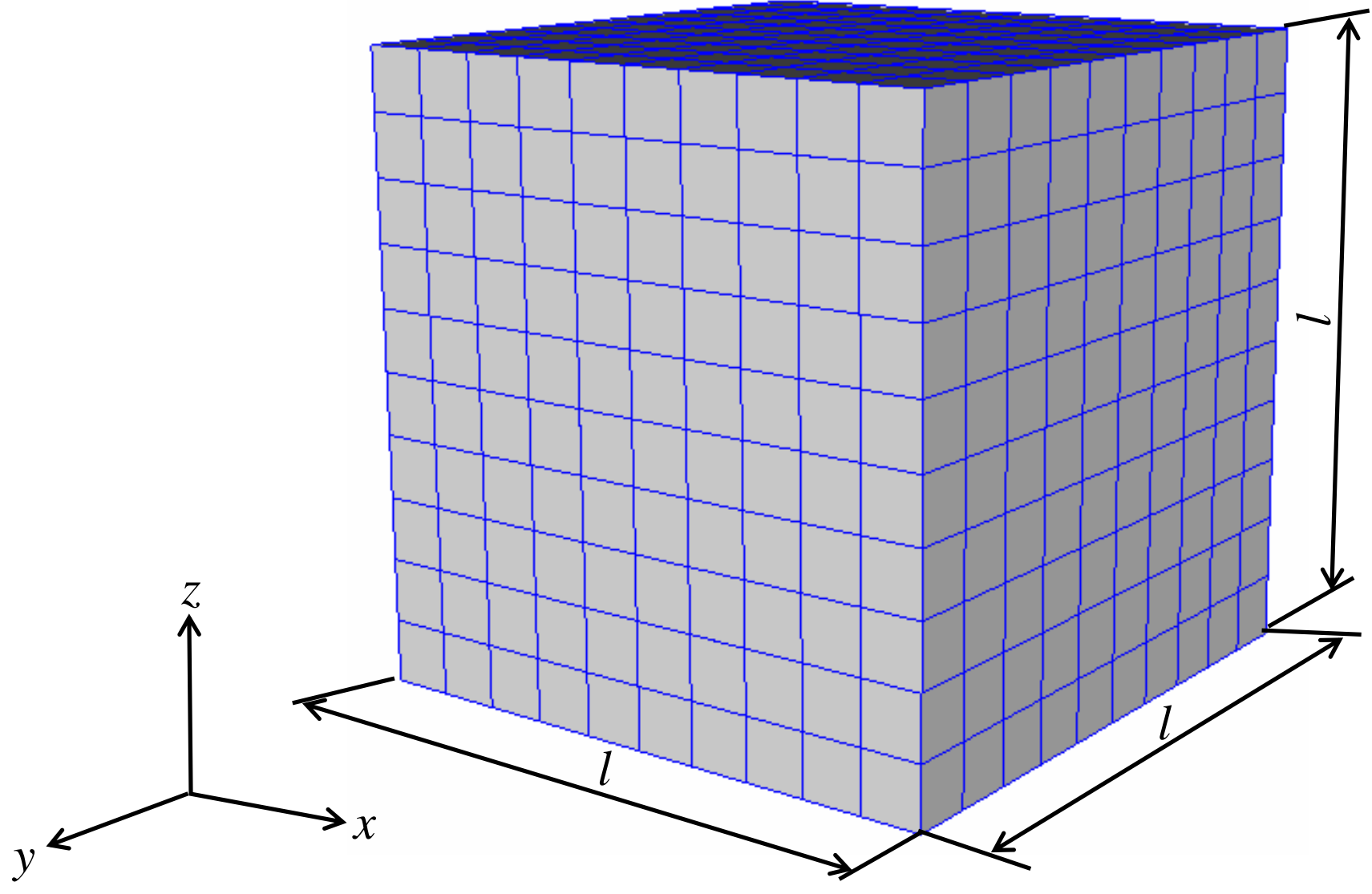

Fig. 1 Finite-element model used in the simulation. 
TD

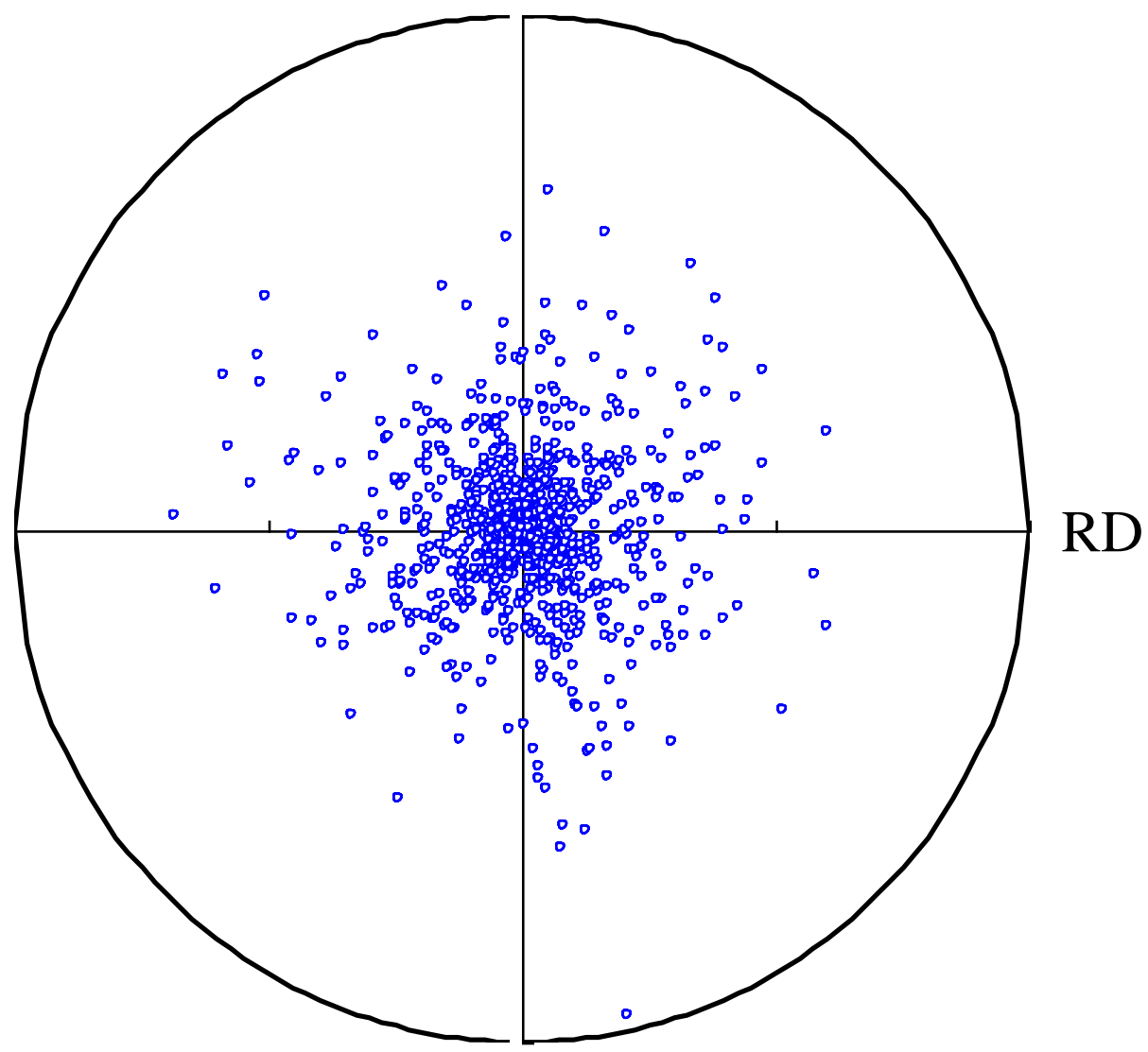

Fig. 2 (0001) pole figure used in the simulation model with rolling texture. 


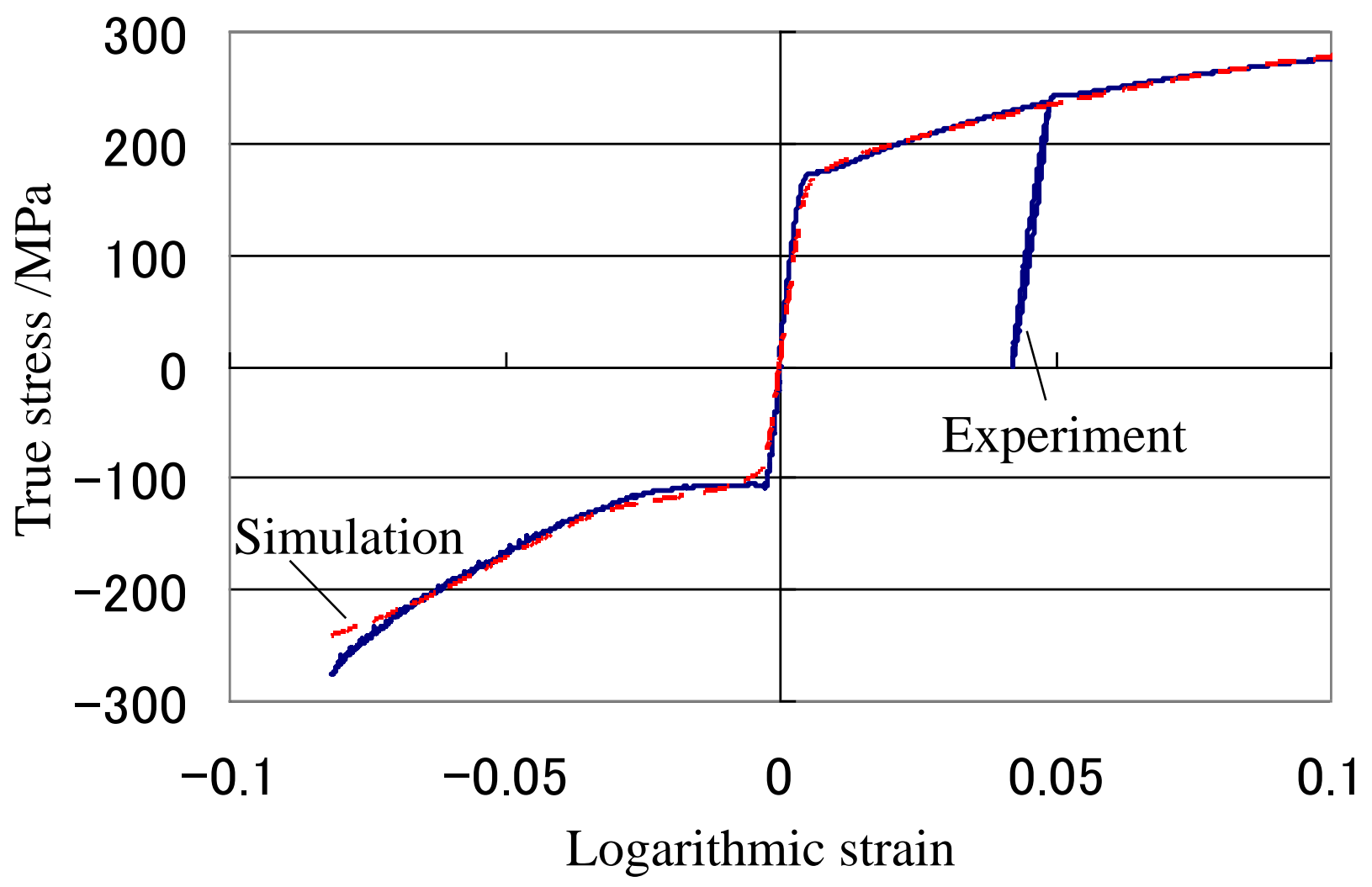

Fig. 3 Stress-strain curves under uniaxial tension and compression obtained by experiment and simulation. 


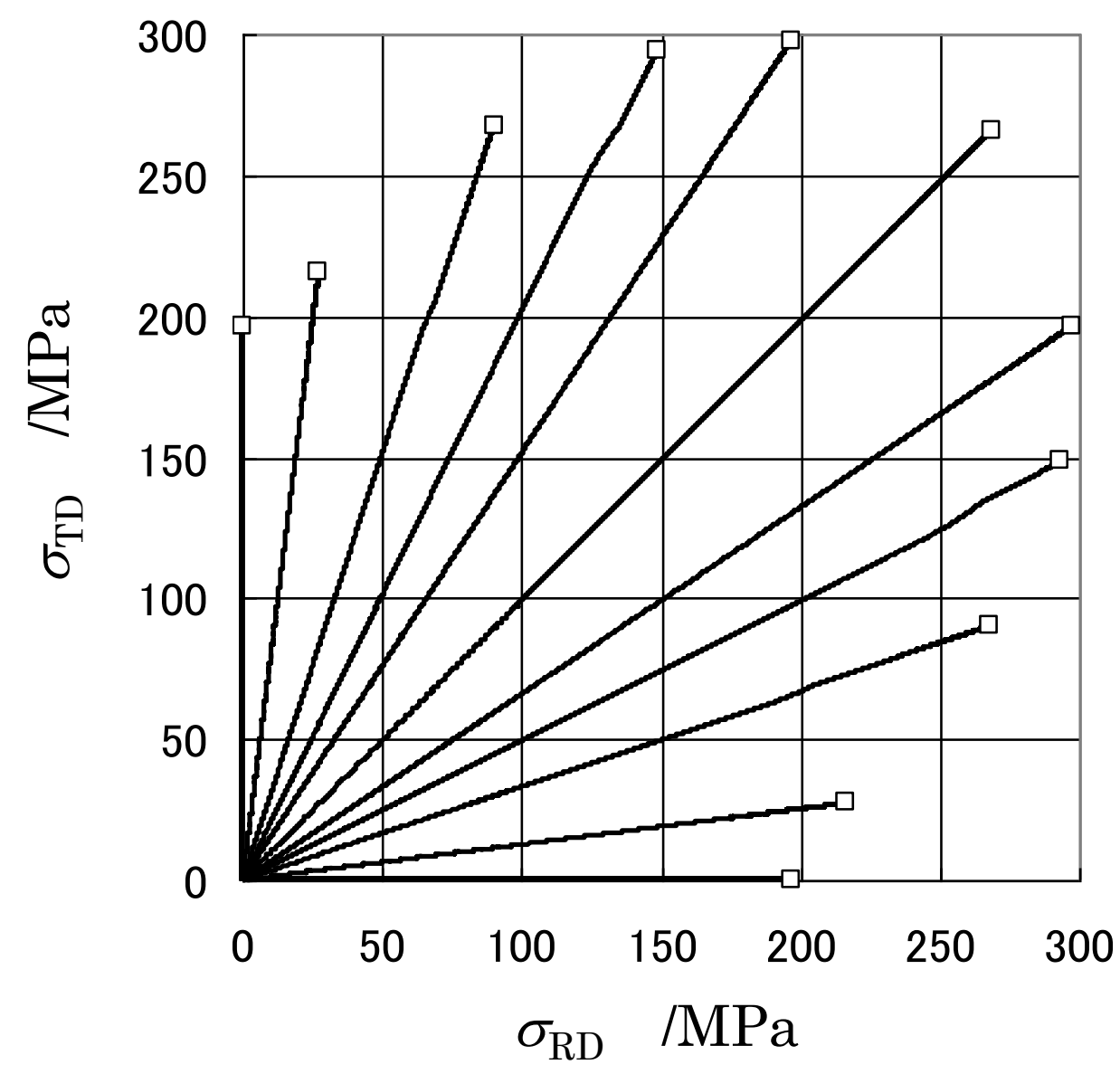

Fig. 4 Stress paths during deformations up to $\varepsilon_{R D}^{p}=1.56 \%$. 
(a) 300

250

$\sum^{\text {Ð }} \begin{aligned} & 200 \\ & 150\end{aligned}$

苗

150

50

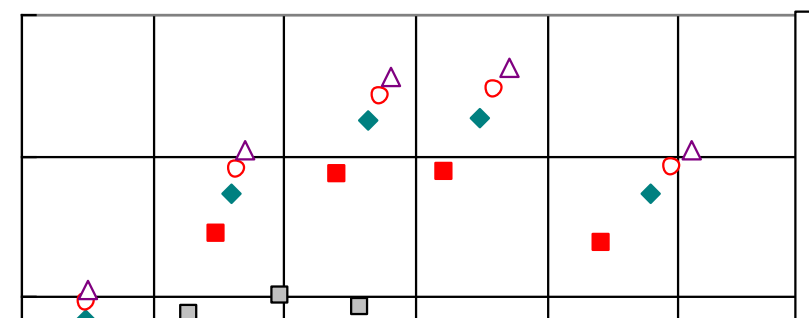

$\diamond 0.08 \%$

$\Delta .14 \%$

* $0.16 \%$

$0.20 \%$

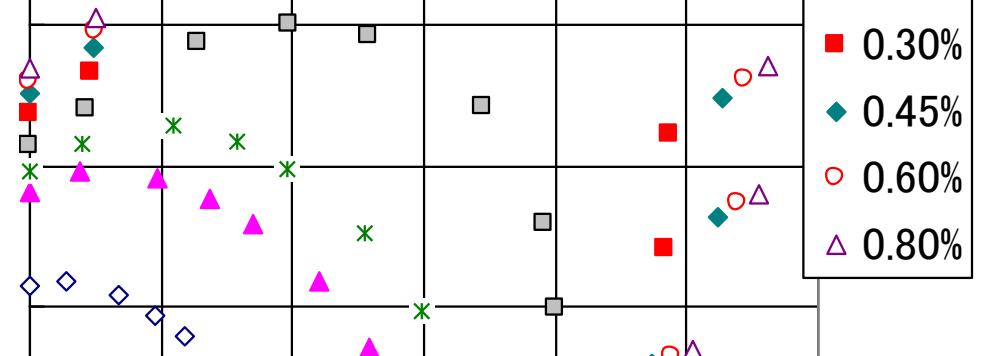

0

$\begin{array}{lllllll}0 & 50 & 100 & 150 & 200 & 250 & 300\end{array}$

$\sigma_{\mathrm{RD}} / \mathrm{MPa}$

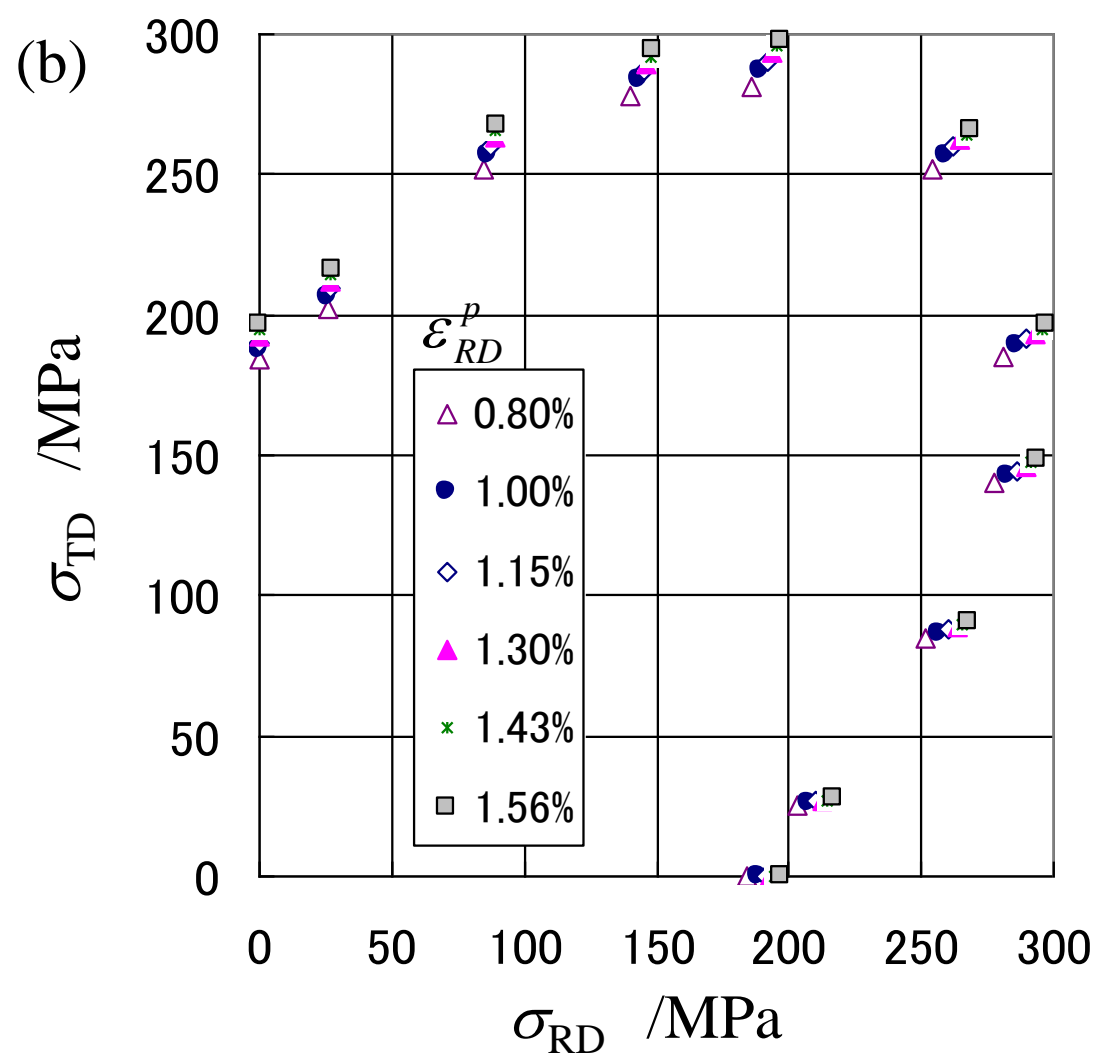

Fig. 5 Contours of plastic work obtained from simulation.

(a) $0.08 \% \leq \varepsilon_{R D}^{p} \leq 0.8 \%$ and (b) $0.8 \% \leq \varepsilon_{R D}^{p} \leq 1.56 \%$ 

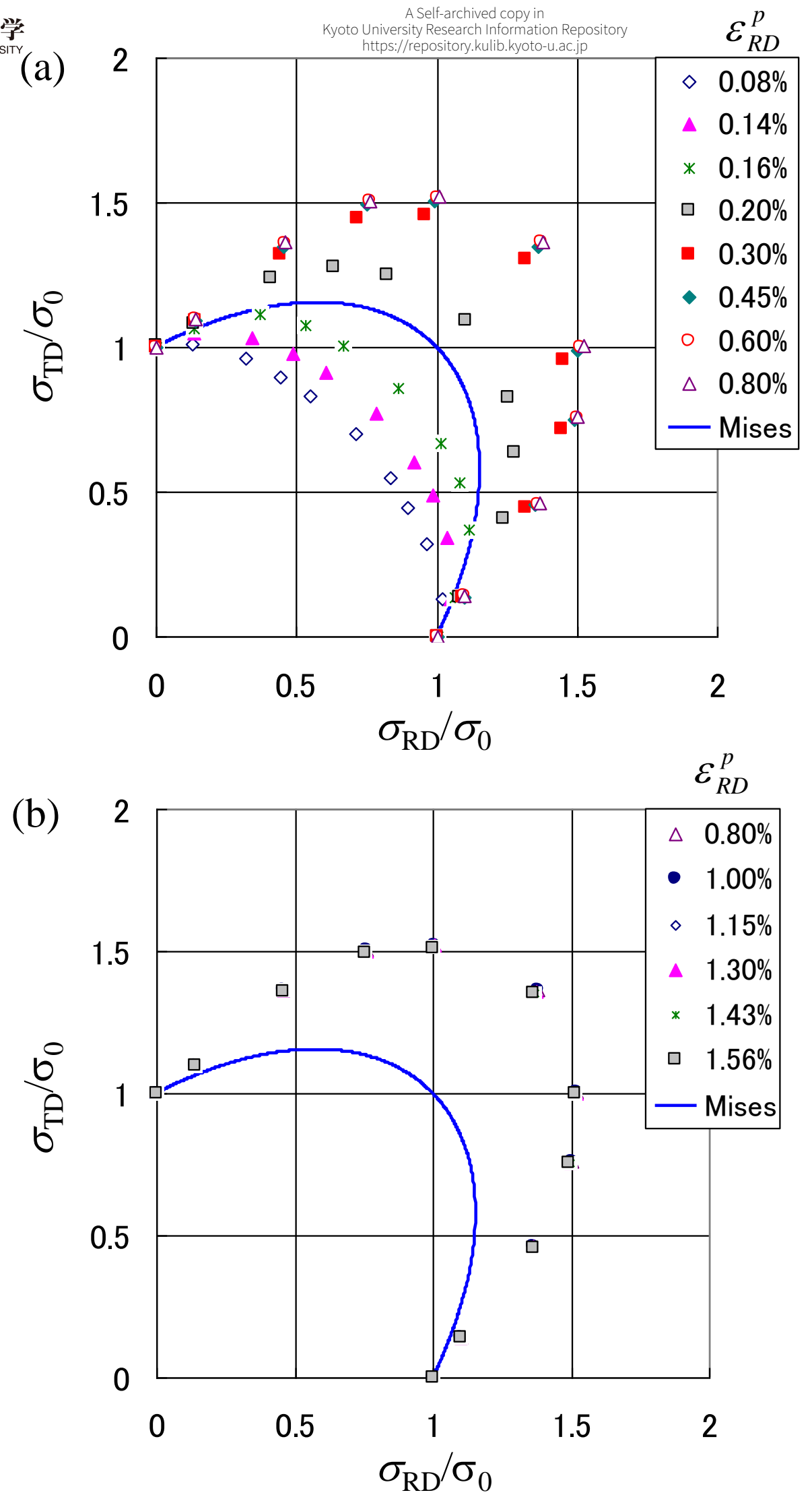

Fig. 6 Normalized contours of plastic work obtained from simulation.

(a) $0.08 \% \leq \varepsilon_{R D}^{p} \leq 0.8 \%$ and (b) $0.8 \% \leq \varepsilon_{R D}^{p} \leq 1.56 \%$ 

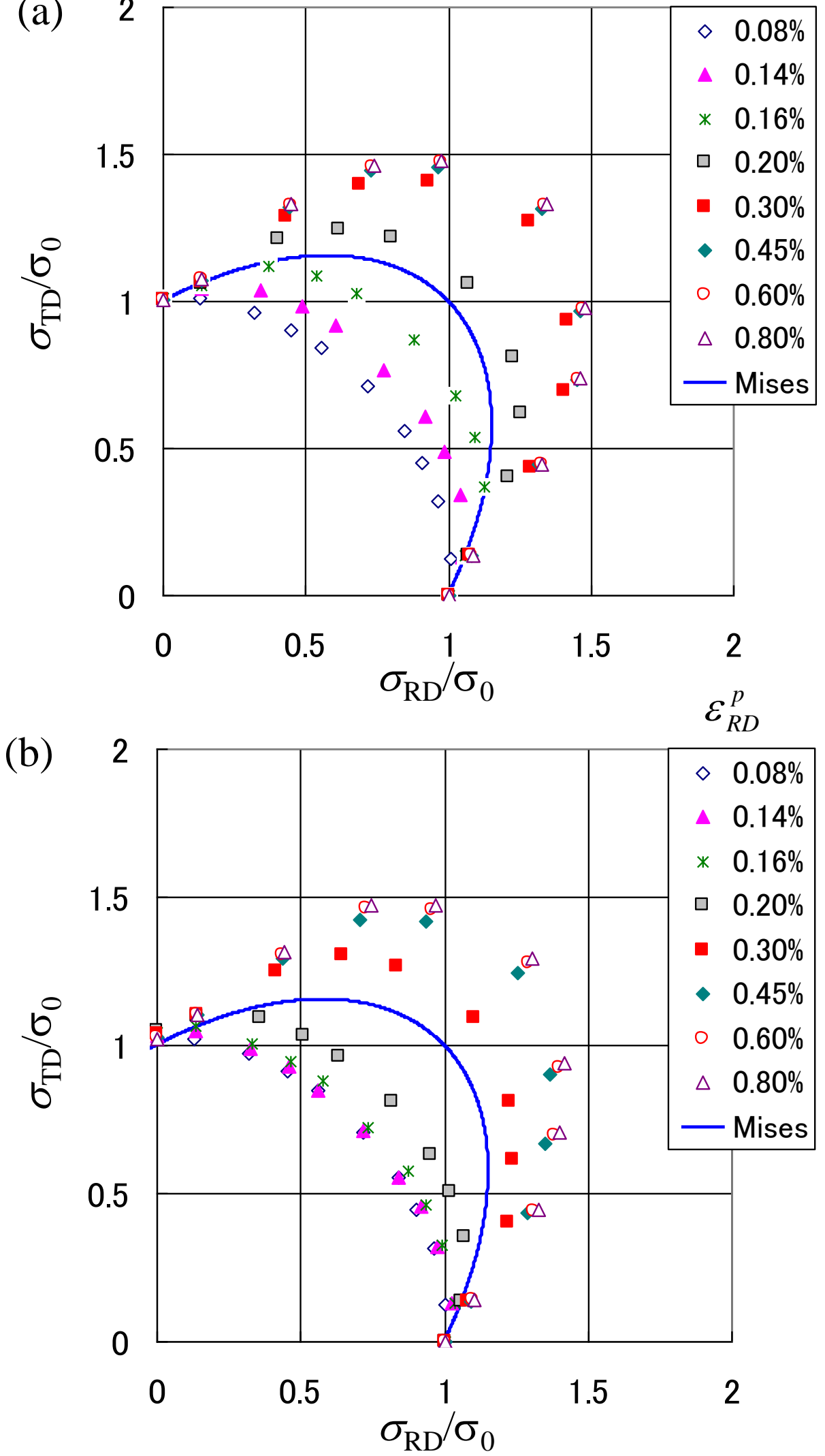

Fig. 7 Normalized contours of plastic work obtained using the two other finite-element models. (a) Seven uniform eight-node brick elements and (b) seven uniform twenty-node brick elements. 
(a)

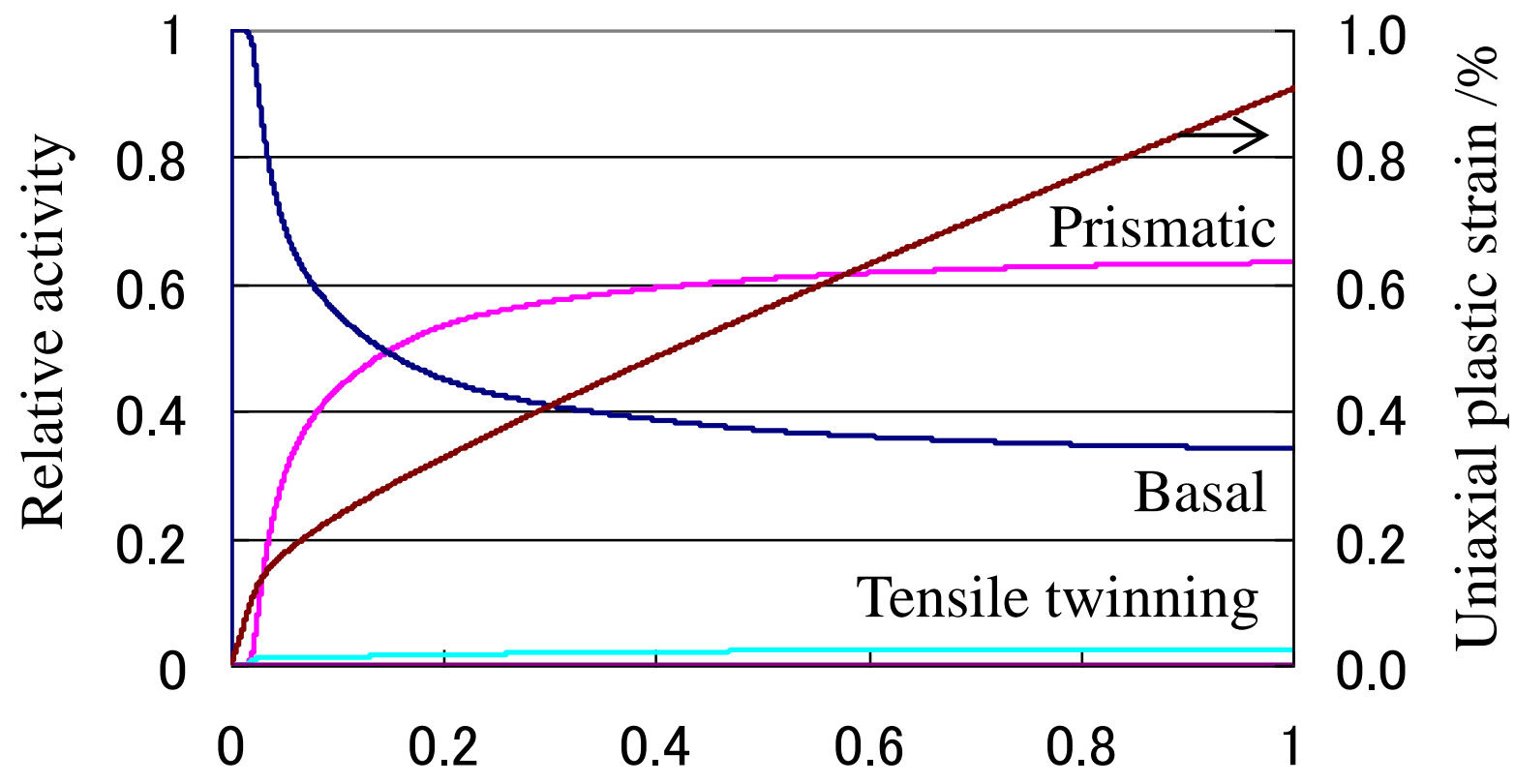

Plastic work $/ \mathrm{MJ}^{*} \mathrm{~m}^{-3}$

(b)

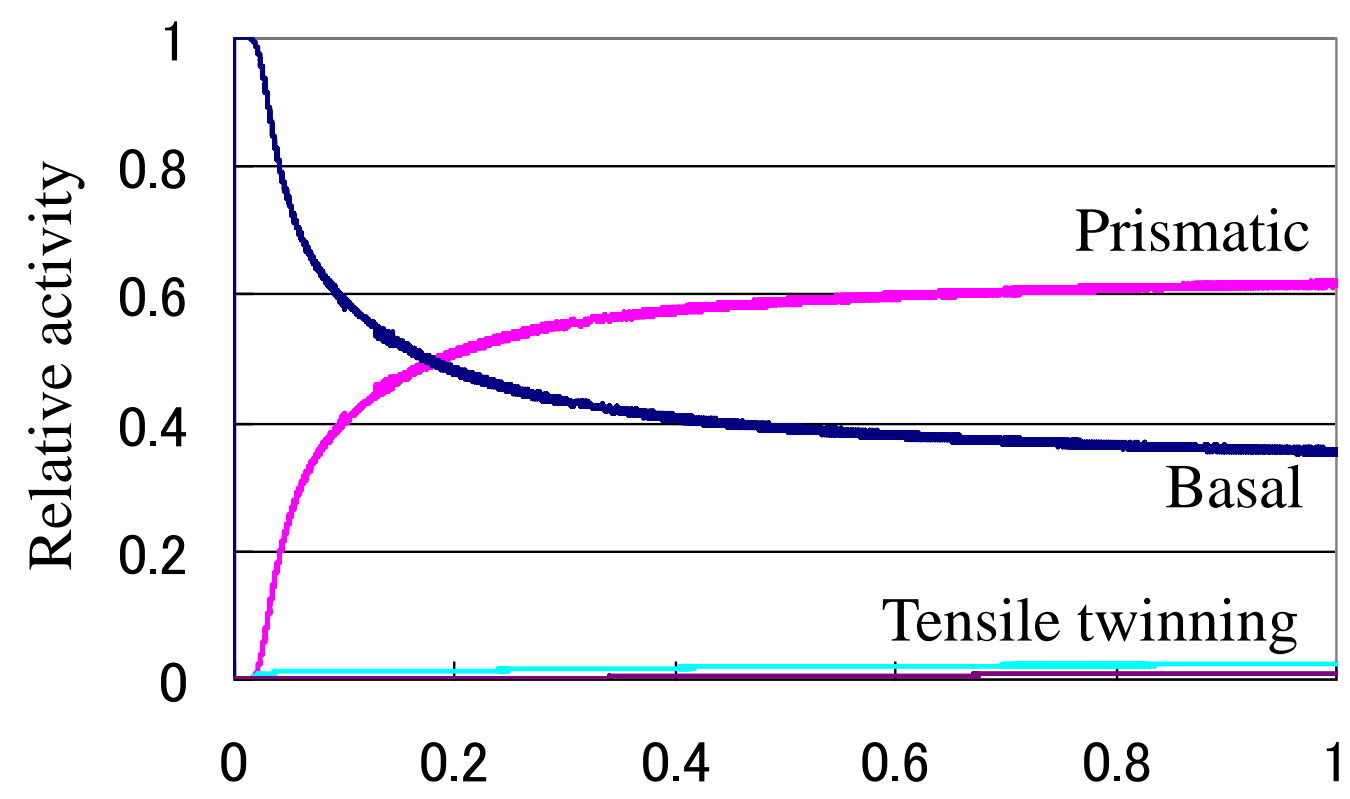

Plastic work $/ \mathrm{MJ}^{*} \mathrm{~m}^{-3}$

Fig. 8 Variations of relative activities as a function of plastic work. (a) $\sigma_{\mathrm{TD}} / \sigma_{\mathrm{RD}}=0$, (b) $\sigma_{\mathrm{TD}} / \sigma_{\mathrm{RD}}=1 / 8$, (c) $\sigma_{\mathrm{TD}} / \sigma_{\mathrm{RD}}=1 / 3$, (d) $\sigma_{\mathrm{TD}} / \sigma_{\mathrm{RD}}=1 / 2$, (e) $\sigma_{\mathrm{TD}} / \sigma_{\mathrm{RD}}=2 / 3$ and (f) $\sigma_{\mathrm{TD}} / \sigma_{\mathrm{RD}}=1$. 
(c)
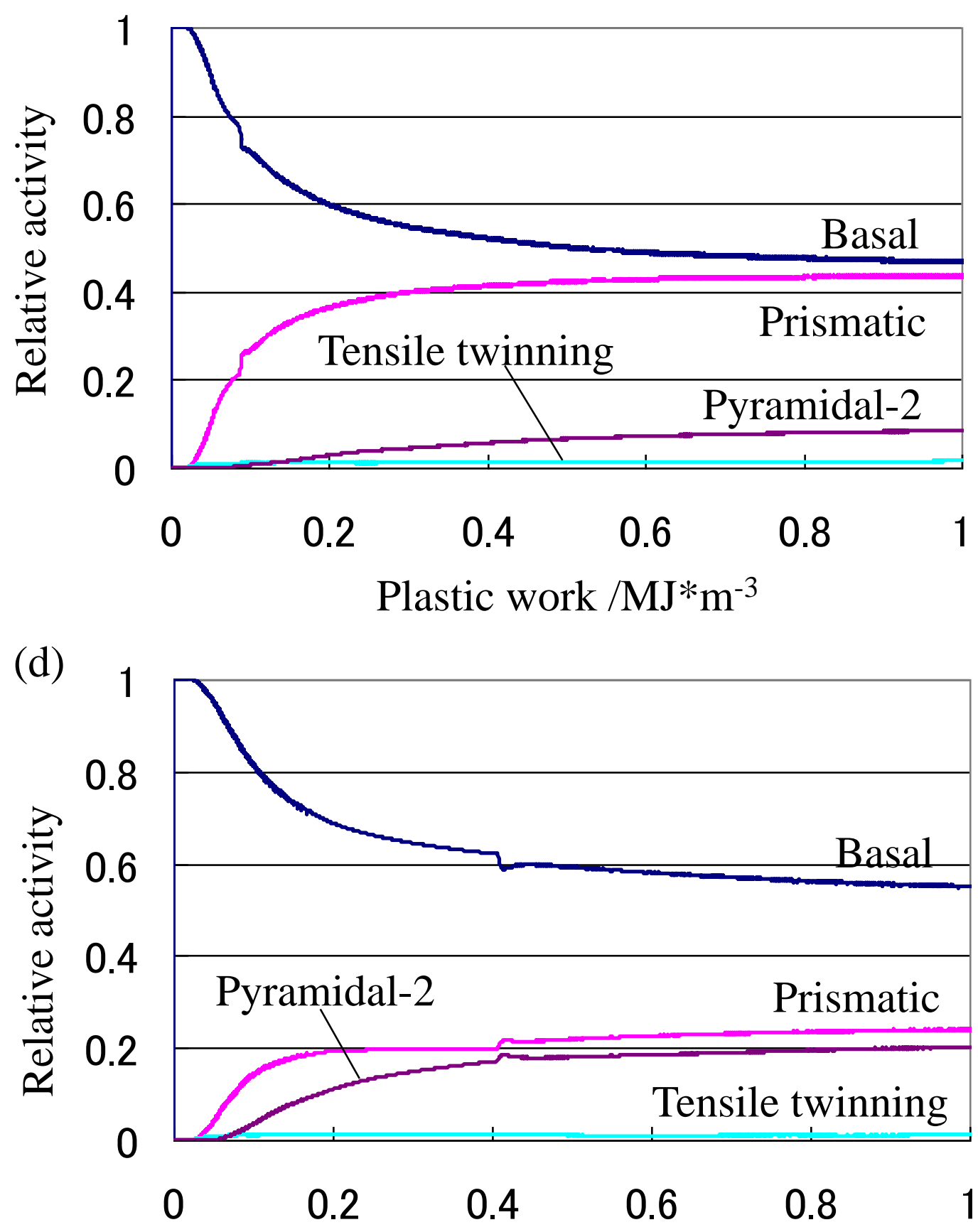

Plastic work $/ \mathrm{MJ}^{*} \mathrm{~m}^{-3}$

Fig. 8 Variations of relative activities as a function of plastic work. (a) $\sigma_{\mathrm{TD}} / \sigma_{\mathrm{RD}}=0$, (b) $\sigma_{\mathrm{TD}} / \sigma_{\mathrm{RD}}=1 / 8$, (c) $\sigma_{\mathrm{TD}} / \sigma_{\mathrm{RD}}=1 / 3$, (d) $\sigma_{\mathrm{TD}} / \sigma_{\mathrm{RD}}=1 / 2$, (e) $\sigma_{\mathrm{TD}} / \sigma_{\mathrm{RD}}=2 / 3$ and (f) $\sigma_{\mathrm{TD}} / \sigma_{\mathrm{RD}}=1$. 
(e)

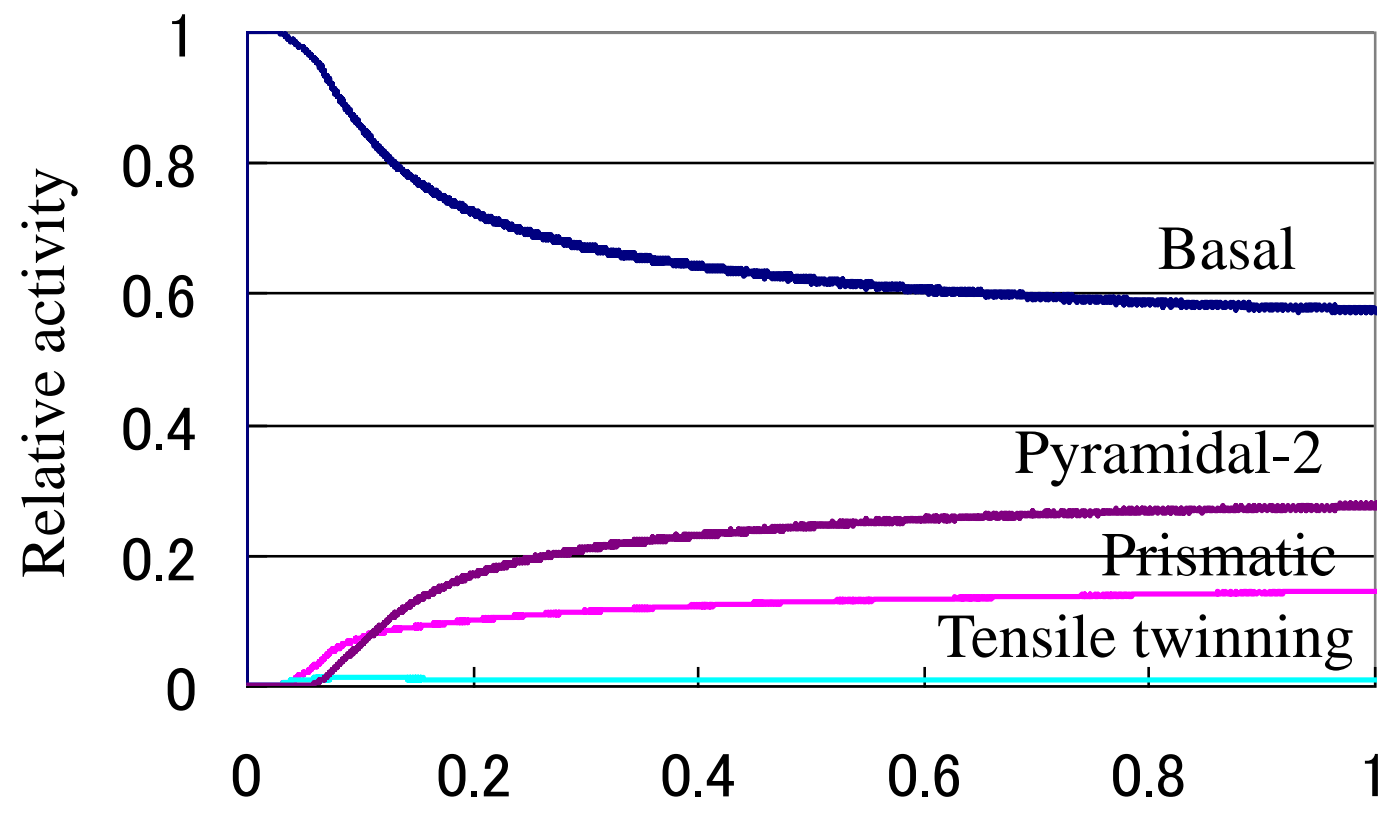

Plastic work $/ \mathrm{MJ}^{*} \mathrm{~m}^{-3}$

(f)

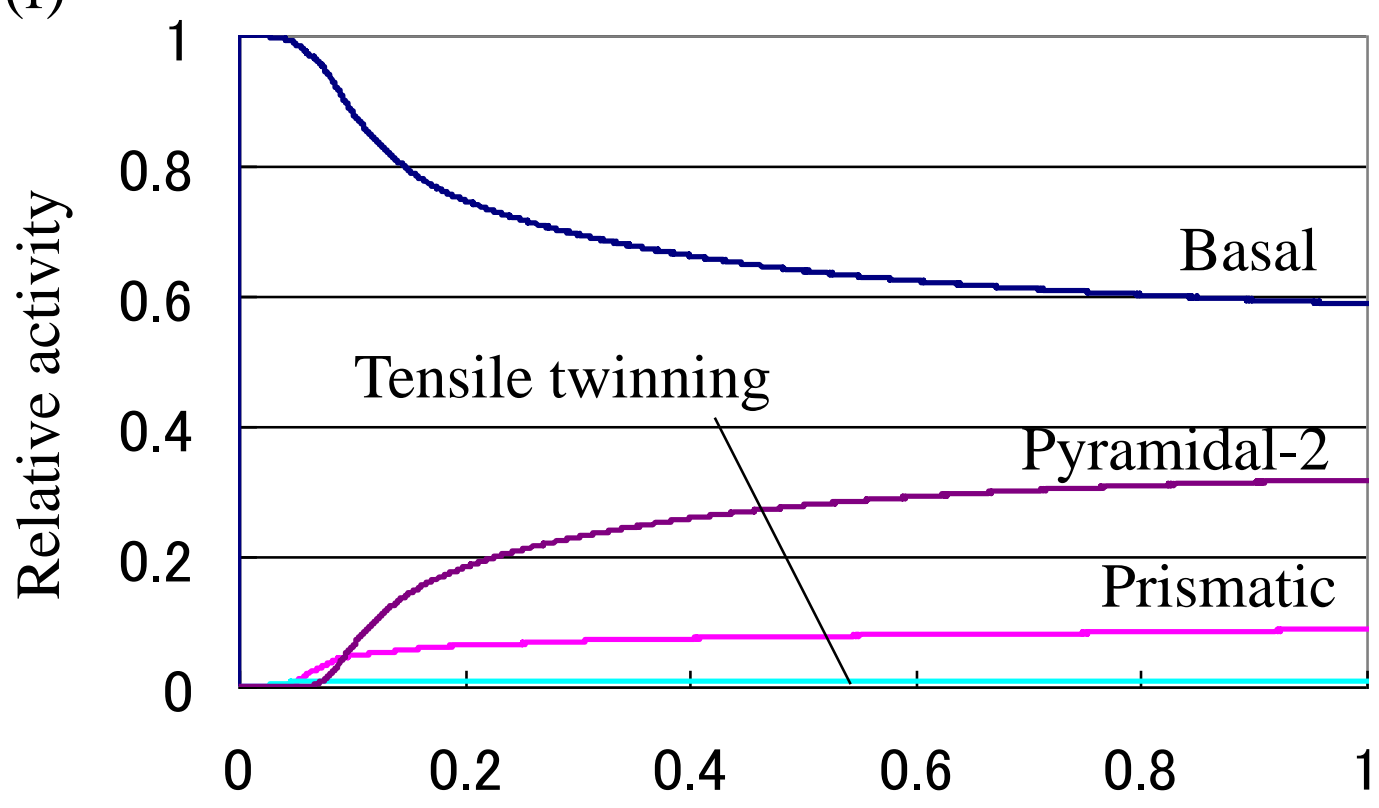

Plastic work $/ \mathrm{MJ}^{*} \mathrm{~m}^{-3}$

Fig. 8 Variations of relative activities as a function of plastic work. (a) $\sigma_{\mathrm{TD}} / \sigma_{\mathrm{RD}}=0$, (b) $\sigma_{\mathrm{TD}} / \sigma_{\mathrm{RD}}=1 / 8$, (c) $\sigma_{\mathrm{TD}} / \sigma_{\mathrm{RD}}=1 / 3$, (d) $\sigma_{\mathrm{TD}} / \sigma_{\mathrm{RD}}=1 / 2$, (e) $\sigma_{\mathrm{TD}} / \sigma_{\mathrm{RD}}=2 / 3$ and (f) $\sigma_{\mathrm{TD}} / \sigma_{\mathrm{RD}}=1$. 
(a)

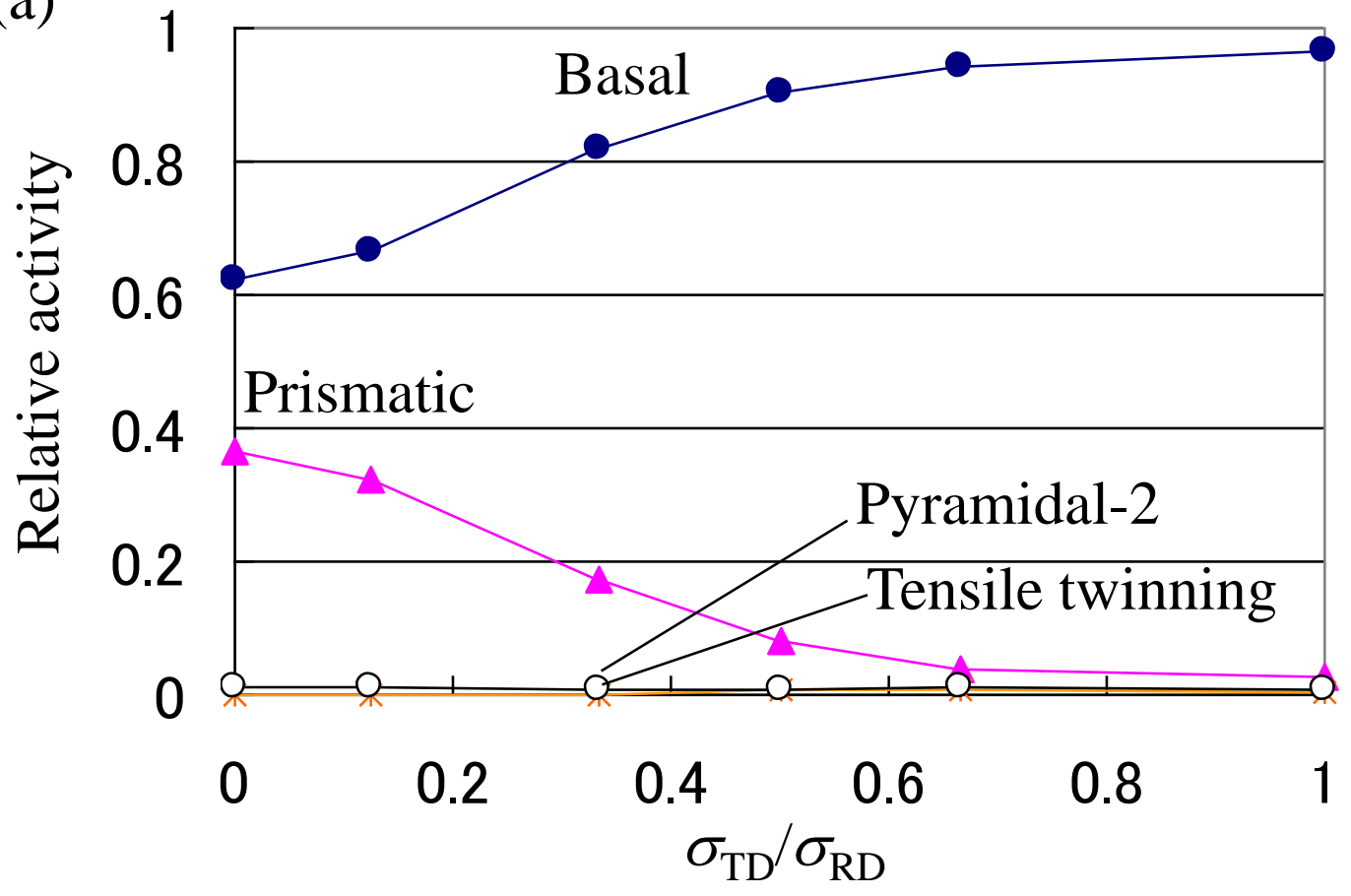

(b)

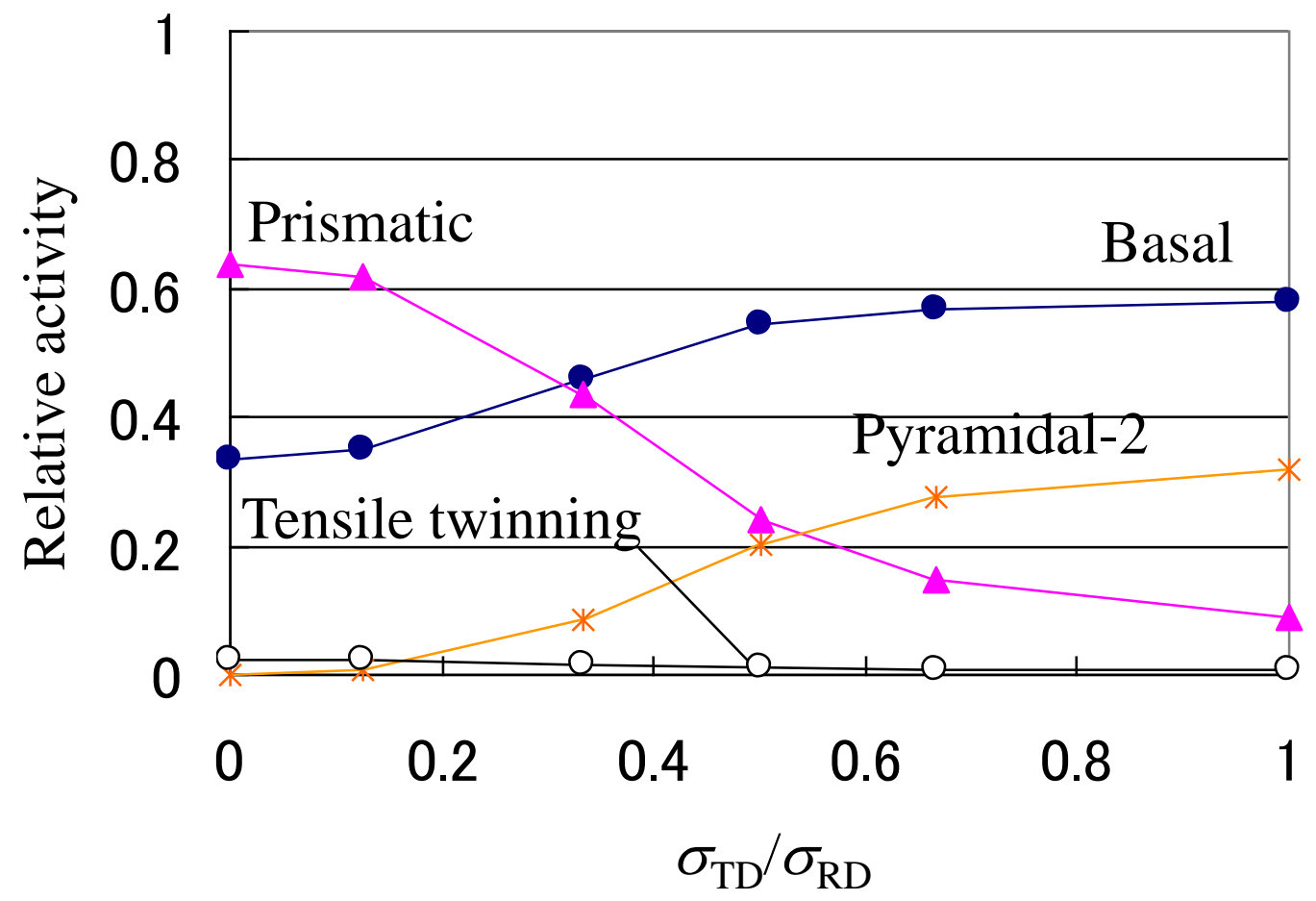

Fig. 9 Correlations between relative activity and biaxial stress ratio.

(a) $0.08 \% \leq \varepsilon_{R D}^{p} \leq 0.8 \%$ and (b) $0.8 \% \leq \varepsilon_{R D}^{p} \leq 1.56 \%$ 


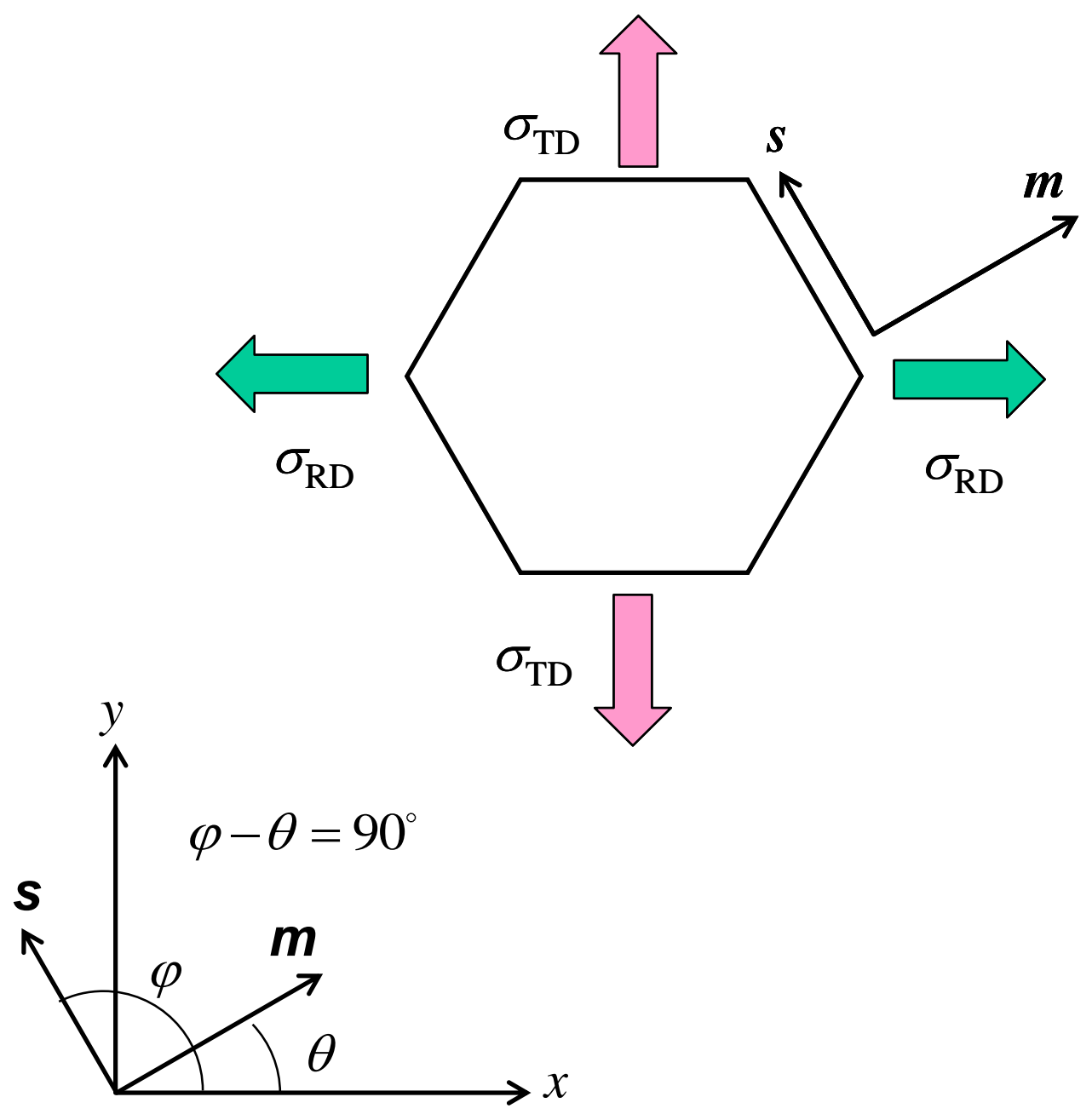

Fig. 10 Simple analytical model of HCP crystal structure on which biaxial stresses are acting. 
Table 1. Plane normal and slip direction vectors of slip and twinning systems used in the present study.

Slip/twinning plane

Basal

Prismatic

Pyramidal-2 $\quad\left\{\begin{array}{llll}1 & 1 & \overline{2} & 2\end{array}\right\}$

Twinning $\quad\left\{\begin{array}{llll}1 & 0 & \overline{1} & 2\end{array}\right\}$
Slip direction/shear direction due to twinning

$\left\langle\begin{array}{llll}1 & 1 & \overline{2} & 0\end{array}\right\rangle$

$\left\langle\begin{array}{llll}1 & 1 & \overline{2} & 0\end{array}\right\rangle$

$\left\langle\begin{array}{llll}1 & 1 & \overline{2} & 3\end{array}\right\rangle$

$\left\langle\begin{array}{llll}1 & 0 & \overline{1} & 1\end{array}\right\rangle$ 
Table 2. Latent-hardening parameters $q_{\alpha \beta}$ used in the present study (Graff et al. [25]).

\begin{tabular}{ccccc}
\hline & Basal & Prismatic & Pyramidal-2 & Twinning \\
Basal & 0.2 & 0.5 & 0.5 & 0.5 \\
Prismatic & 0.2 & 0.2 & 0.2 & 0.5 \\
Pyramidal-2 & 1.0 & 1.0 & 0.2 & 0.25 \\
Twinning & 1.0 & 1.0 & 0.2 & 0.25 \\
\hline
\end{tabular}


Table 3. Calibrated material parameters in Eqs. (4) and (5).

\begin{tabular}{ccccc}
\hline & Basal & Prismatic & Pyramidal-2 & Twinning \\
$\tau_{0}$ & 10 & 85 & 160 & 48 \\
$\tau_{\infty}$ & - & 260 & 360 & - \\
$h_{0}$ & 10 & 600 & 750 & 150 \\
\hline
\end{tabular}


Table 4. The Schmid factors $m_{x} s_{x}$ and $m_{y} s_{y}$ in eq.(10) of the pyramidal-2 slip systems.

\begin{tabular}{ccccccc} 
& $(2 \overline{1} \overline{1} 2)$ & $(11 \overline{2} 2)$ & $(\overline{1} \overline{1} 22)$ & $(1 \overline{2} 12)$ & $(\overline{1} 2 \overline{1} 2)$ & $(\overline{2} 112)$ \\
\hline$m_{x} s_{x}$ & -0.1116 & 0.1116 & -0.1116 & -0.4465 & 0.4465 & 0.1116 \\
$m_{y} s_{y}$ & -0.3349 & 0.3349 & -0.3349 & 0.0000 & 0.0000 & 0.3349 \\
\hline
\end{tabular}

